\title{
Cohomology pairings on singular quotients in geometric invariant theory
}

\author{
Lisa C. Jeffrey \\ Mathematics Department, University of Toronto \\ Toronto, ON M5S 3G3, Canada, ${ }^{*}$ \\ Young-Hoon Kiem \\ Department of Mathematics, Stanford University \\ Stanford, CA 94305-2060, USA \\ Frances Kirwan \\ Balliol College, Oxford OX1 3BJ, UK \\ and \\ Jonathan Woolf \\ Christ's College, Cambridge, CB2 3BU, UK. ${ }^{\dagger}$
}

May 2002

\section{Introduction}

Let $M / / G$ denote the quotient in the sense of Mumford's geometric invariant theory [34] of a nonsingular connected complex projective variety $M$ by an action of a connected complex reductive group $G$ which is linear with respect to an ample line bundle $L$ on $M$. Such quotients often appear as moduli spaces or as compactifications of moduli spaces in algebraic geometry, and

\footnotetext{
${ }^{*}$ The author acknowledges support from NSERC and the Alfred P. Sloan Foundation.

${ }^{\dagger}$ Partially supported by the Seggie-Brown Research Fellowship at the University of Edinburgh.
} 
their topology has been studied for many years, stimulated in particular by the inspiring work of Atiyah and Bott [2] in the early 1980s and Witten [39] a decade later. In 20, (see also [14, 21, 29, 30, 31, 39]) formulas were obtained for the intersection pairings of cohomology classes of complementary dimensions in $H^{*}(M / / G)$ under the assumption that every semistable point of $M$ is stable. In that case the quotient $M / / G$ has only orbifold singularities and its cohomology with complex coefficients behaves much like that of a nonsingular projective variety; in particular its intersection cohomology $I H^{*}(M / / G)$ is the same as its ordinary cohomology $H^{*}(M / / G)$. (Intersection cohomology is defined with respect to the middle perversity throughout this paper, and all cohomology and homology groups have complex coefficients.) In this paper we shall give formulas (see Theorem 8.4) for the pairings of intersection cohomology classes of complementary dimensions in the intersection cohomology $I H^{*}(M / / G)$ of geometric invariant theoretic quotients $M / / G$ for which semistability is not necessarily the same as stability (although we make some weaker assumptions on the action). We also give formulas for intersection pairings on resolutions of singularities (or more precisely partial resolutions, since orbifold singularities are allowed) of the quotients $M / / G$ (see Proposition 8.1).

Let $K$ be a maximal compact subgroup of the reductive group $G$, and let $\mathbf{k}$ denote its Lie algebra. Then using the given linearization of the $G$-action on $M$ we can choose a $K$-invariant Kähler form $\omega$ on $M$, and the action of $K$ on $M$ is Hamiltonian with respect to the symplectic structure given by $\omega$; i.e. there exists a moment map $\mu: M \rightarrow \mathbf{k}^{*}$ for the action (see for example Chapter 2 of [25]). The inclusion of $\mu^{-1}(0)$ in the set $M^{s s}$ of semistable points of $M$ induces a homeomorphism from the Marsden-Weinstein reduction, or symplectic quotient, $\mu^{-1}(0) / K$ to the geometric invariant theoretic quotient $M / / G$ (25] 8.14). The condition that semistability equals stability is equivalent to the condition that 0 is a regular value of the moment map, and implies that the cohomology $H^{*}(M / / G)$ of the quotient $M / / G$ is naturally isomorphic to the equivariant cohomology $H_{K}^{*}\left(\mu^{-1}(0)\right)$ of $\mu^{-1}(0)$ (recall that we are working with cohomology with complex coefficients). The restriction map $H_{K}^{*}(M) \rightarrow H_{K}^{*}\left(\mu^{-1}(0)\right)$ is surjective (25] 5.4), and, as a module over the equivariant cohomology of a point, which we write as $H_{K}^{*}$, the equivariant cohomology $H_{K}^{*}(M)$ of $M$ is just the tensor product of its ordinary cohomology $H^{*}(M)$ and $H_{K}^{*}$ (25] 5.8). When semistability coincides with stability, the composition of the restriction map $H_{K}^{*}(M) \rightarrow H_{K}^{*}\left(\mu^{-1}(0)\right)$ and the isomorphism $H_{K}^{*}\left(\mu^{-1}(0)\right) \rightarrow H^{*}(M / / G)$ gives us a natural surjective ring homomorphism

$$
\kappa_{M}: H_{K}^{*}(M) \rightarrow H^{*}(M / / G) .
$$

The residue formula of [20] is a formula for pairings of cohomology classes of complementary dimensions in $M / / G$ in terms of equivariant cohomology classes in $M$ which represent them via this surjection $\kappa_{M}$, in the case when semistability equals stability. This formula was obtained from a version 
of Witten's nonabelian localization principle 39] for compact Hamiltonian group actions. For $\epsilon>0$ and $\zeta$ a formal $K$-equivariant cohomology class on $M$ given by a sum $\zeta=\sum_{j \geq 0} \zeta_{j}$ where $\zeta_{j} \in H_{K}^{j}(M)$, Witten defines an integral $I^{\epsilon}(\zeta)$, which depends on choosing a fixed invariant inner product $<$, $>$ on $\mathbf{k}$. If we represent elements of $H_{K}^{*}(M)$ by polynomial functions on $\mathbf{k}$ with values in the De Rham complex $\Omega^{*}(M)$, this integral is

$$
I^{\epsilon}(\zeta)=\frac{1}{(2 \pi)^{s} \operatorname{vol}(K)} \int_{X \in \mathbf{k}}[d X] e^{-\epsilon<X, X>/ 2} \int_{M} \zeta(X)
$$

where $\int_{M}: H_{K}^{*}(M) \rightarrow H_{K}^{*}$ is the pushforward map given by integration over $M$, the measure $[d X]$ on $\mathbf{k}$ is induced by the fixed inner product, $\operatorname{vol}(K)$ is the integral of the induced volume form on $K$ and $s$ is the dimension of $K$. When $\zeta$ is of the form

$$
\zeta=\eta e^{i \bar{\omega}}
$$

where $\eta \in H_{K}^{*}(M)$ and $\bar{\omega}$ is the extension $\bar{\omega}=\omega+\mu$ of the symplectic form $\omega$ to an equivariantly closed differential form on $M$, Witten expresses this integral as a sum of local contributions. If we assume for simplicity that the stabilizer in $K$ of a generic point of $\mu^{-1}(0)$ is trivial, then one of these local contributions reduces to the evaluation

$$
\kappa_{M}(\zeta) e^{\epsilon \Theta}[M / / G]
$$

of the fundamental class $[M / / G]$ against the product $\kappa_{M}(\zeta) e^{\epsilon \Theta}$, where $\kappa_{M}(\zeta)$ is the cohomology class on $M / / G$ induced by $\zeta$ and $\Theta$ is the image under the natural map

$$
H_{K}^{*} \rightarrow H_{K}^{*}\left(\mu^{-1}(0)\right) \cong H^{*}(M / / G)
$$

of a distinguished class in $H_{K}^{4}$. In fact if we identify $H_{K}^{*}$ in the natural way with the space of $K$-invariant polynomials on $\mathbf{k}$ then $\Theta$ is the class represented by $X \mapsto-\frac{1}{2}\langle X, X>$. This local contribution is thus a polynomial in $\epsilon$, whereas the other terms in Witten's expression for $I^{\epsilon}(\zeta)$ as a sum of local contributions all tend to zero exponentially fast as $\epsilon$ tends to 0 .

Of course if the degree of $\eta \in H_{K}^{*}(M)$ is equal to the real dimension of $M / / G$, then for $\zeta=\eta e^{i \bar{\omega}}$ we have

$$
\kappa_{M}(\zeta) e^{\epsilon \Theta}[M / / G]=\kappa_{M}(\eta) e^{i \omega_{0}+\epsilon \Theta}[M / / G]=\kappa_{M}(\eta)[M / / G]
$$

where $\omega_{0}$ is the induced symplectic form on $M / / G$; note that $\bar{\omega}=\omega$ on $\mu^{-1}(0)$ since $\mu$ vanishes there. Also $\kappa_{M}$ is a ring homomorphism, so if $\kappa_{M}(\alpha)$ and $\kappa_{M}(\beta)$ are cohomology classes of complementary degrees in $M / / G$ then their intersection pairing is given by $\kappa_{M}(\alpha \beta)[M / / G]$. Thus the behaviour as $\epsilon \rightarrow 0$ of the integrals $I^{\epsilon}\left(\alpha \beta e^{i \bar{\omega}}\right)$ determines the intersection pairings of cohomology classes $\kappa_{M}(\alpha)$ and $\kappa_{M}(\beta)$ of complementary degrees in $M / / G$. Moreover since $M / / G$ has at worst orbifold singularities, its cohomology $H^{*}(M / / G)$ satisfies Poincaré duality and so these pairings in principle determine the kernel of 
the surjection $\kappa_{M}: H_{K}^{*}(M) \rightarrow H^{*}(M / / G)$, and hence the ring structure of $H^{*}(M / / G)$ given the ring structure of $H_{K}^{*}(M)$.

In 20] the integral $I^{\epsilon}\left(\eta e^{i \bar{\omega}}\right)$ is rewritten as an integral over the Lie algebra $\mathbf{t}$ of a maximal torus $T$ of $K$. The localization theorem for compact abelian actions proved by Berline and Vergne [6] and by Atiyah and Bott [3] is used to decompose this integral as a sum of terms indexed by the set $\mathcal{F}$ of components of the fixed point set $M^{T}$ of $T$ on $M$. This leads to a formula (the residue formula, Theorem 8.1 of [20]; see Theorem 3.1 of [22] for a corrected version) for $\kappa_{M}(\eta) e^{i \omega_{0}}[M / / G]$. In fact there is no need to include the factor of $i$ here, so we shall follow the conventions of 22 and omit it. If $n_{0}$ is the order of the stabilizer in $K$ of a generic point of $\mu^{-1}(0)$ the residue formula then is

$$
\kappa_{M}(\eta) e^{\omega_{0}}[M / / G]=\frac{n_{0}(-1)^{s+n_{+}}}{|W| \operatorname{vol}(T)} \operatorname{res}\left(\mathcal{D}(X)^{2} \sum_{F \in \mathcal{F}_{+}} \int_{F} \frac{i_{F}^{*}\left(\eta e^{\bar{\omega}}\right)(X)}{e_{F}(X)}[d X]\right)
$$

where $\operatorname{vol}(T)$ and $[d X]$ are the volume of $T$ and the measure on its Lie algebra $\mathbf{t}$ induced by the restriction to $\mathbf{t}$ of the fixed inner product on $\mathbf{k}$, while $W$ is the Weyl group of $K$, the polynomial function $\mathcal{D}(X)$ of $X \in \mathbf{t}$ is the product of the positive $\operatorname{roots}^{1}$ of $K$ and $n_{+}=(s-l) / 2$ is the number of those positive roots; as before, $s$ is the dimension of $K$ and $l$ is the dimension of $T$. Also $\mathcal{F}_{+}$is a subset of $\mathcal{F}$ consisting of those components $F$ of the fixed point set $M^{T}$ on which the constant value taken by the $T$-moment map $\mu_{T}: M \rightarrow \mathbf{t}^{*}$ (which is the composition of $\mu: M \rightarrow \mathbf{k}^{*}$ with the natural map $\mathbf{k}^{*} \rightarrow \mathbf{t}^{*}$ ) lies in a certain cone in $\mathbf{t}^{*}$ with its vertex at 0 , and if $F \in \mathcal{F}$ then $i_{F}: F \rightarrow M$ is the inclusion and $e_{F}$ is the equivariant Euler class of the normal bundle to $F$ in $M$.

In this paper we consider the more general situation where there may be semistable points of $M$ which are not stable (or equivalently 0 is not a regular value of $\mu$ ); we assume only that there do exist some stable points (or equivalently that there exist some points in $\mu^{-1}(0)$ where the derivative of $\mu$ is surjective). Then there is no longer a natural surjection from $H_{K}^{*}(M)$ to $H^{*}(M / / G)$, and since $M / / G$ is in general singular (with singularities more serious than orbifold singularities) its cohomology $H^{*}(M / / G)$ may not satisfy Poincaré duality. However even for singular complex projective varieties, the intersection cohomology groups defined by Goresky and MacPherson [11, 12. satisfy Poincaré duality, as well as the other properties of the cohomology groups of nonsingular complex projective varieties known collectively as the Kähler package. Moreover the intersection cohomology $I H^{*}(M / / G)$ of the quotient $M / / G$ is a direct summand of the ordinary cohomology of any resolution of singularities of $M / / G$; this is a special case of the decomposition

\footnotetext{
${ }^{1}$ In this paper, as in $\left[22\right.$, we adopt the convention that weights $\beta \in \mathbf{t}^{*}$ send the integer lattice $\Lambda^{I}=\operatorname{ker}(\exp : \mathbf{t} \rightarrow T)$ to $\mathbb{Z}$ rather than to $2 \pi \mathbb{Z}$, and that the roots of $K$ are the nonzero weights of its complexified adjoint action. This is one reason why the constant in the residue formula above differs from that of [20] Theorem 8.1 (see the footnotes on pages $123-5$ of $[22]$ ).
} 
theorem of Beilinson, Bernstein, Deligne and Gabber [4].

There is a canonical procedure (see 26]) for constructing a partial resolution of singularities $\tilde{M} / / G$ of the quotient $M / / G$. This involves blowing $M$ up along a sequence of nonsingular $G$-invariant subvarieties, all contained in the complement $M-M^{s}$ of the set $M^{s}$ of stable points of $M$, to eventually obtain a nonsingular projective variety $\tilde{M}$ with a linear $G$-action, lifting the action on $M$, for which every semistable point of $\tilde{M}$ is stable. Then the quotient $\tilde{M} / / G$ has only orbifold singularities, and the blowdown map $\pi: \tilde{M} \rightarrow M$ induces a birational morphism $\pi_{G}: \tilde{M} / / G \rightarrow M / / G$ which is an isomorphism over the dense open subset $M^{s} / G$ of $M / / G$.

Since we are working with complex coefficients and neglecting torsion, orbifold singularities cause few difficulties and in particular the intersection cohomology $I H^{*}(M / / G)$ of $M / / G$ is a direct summand of the cohomology of its partial resolution of singularities $\tilde{M} / / G$. So we can consider the composition

$$
H_{K}^{*}(M) \rightarrow H_{K}^{*}(\tilde{M}) \rightarrow H^{*}(\tilde{M} / / G) \rightarrow I H^{*}(M / / G)
$$

of maps, of which the first is induced by the blowdown map $\tilde{M} \rightarrow M$, the second is $\kappa_{\tilde{M}}$ (see (1.10) and the third is the projection of $H^{*}(\tilde{M} / / G$ ) onto its direct summand $I H^{*}(M / / G)$. This composition is surjective (see [27, 41]) and in many ways it is a natural generalization of the map $\kappa_{M}: H_{K}^{*}(M) \rightarrow$ $H^{*}(M / / G)$ defined when $M^{s s}=M^{s}$ at (1.1), so we shall call it $\kappa_{M}$ too. Since the inclusion of $I H^{*}(M / / G)$ as a direct summand of $H^{*}(\tilde{M} / / G)$ respects the intersection pairings of classes of complementary dimensions (see [23] Section 6 ), it is reasonable to hope that the residue formula (1.4) can be applied to the quotient $\tilde{M} / / G$ to yield a formula for the intersection pairings of classes $\kappa_{M}(\alpha)$ and $\kappa_{M}(\beta)$ of complementary dimensions in $I H^{*}(M / / G)$.

Unfortunately various complications arise when we try to apply the residue formula (1.4) above to $\tilde{M}$ to obtain pairings on the partial desingularization $\tilde{M} / / G$. In particular, although the construction of $\tilde{M} / / G$ from the linear $G$-action on $M$ is canonical and explicit, the construction of $\tilde{M}$ is not. In fact the procedure given in [26] is to blow up the set $M^{s s}$ of semistable points of $M$ along a sequence of nonsingular $G$-invariant closed subvarieties $V$ of $M^{s s}$, after each blow-up throwing out any points which are not semistable, to eventually arrive at $\tilde{M}^{s s}$ and thus obtain $\tilde{M} / / G=\tilde{M}^{s s} / G$. The variety $\tilde{M}$ itself can be obtained by resolving the singularities of the closures $\bar{V}$ of these subvarieties $V$ and blowing up along their proper transforms, but in practice this is not usually simple. Since the residue formula (1.4) involves the set of components of the fixed point set of the action of the maximal torus $T$ of $K$, applying it directly to $\tilde{M}$ is likely to be very complicated; knowledge of the set of semistable points $\tilde{M}^{s s}$ alone would certainly not be sufficient. Luckily it turns out that there is a simpler way to obtain the pairings.

It is worth observing, however, that when $K$ is itself a compact torus $T$ (or equivalently when the complexification $G=K_{c}$ of $K$ is a complex torus $T_{c}$ ) then most of the difficulties described above disappear. In this case $\tilde{M}$ 
can be obtained from $M$ by blowing up along the components which meet $M^{s s}$ (or equivalently which meet $\mu^{-1}(0)$ ) of the fixed point sets of subtori $T^{\prime}$ of $T$, in decreasing order of the dimension of $T^{\prime}$.

In the general case we can make use of the key observation due to S. Martin 29, 30] and independently to Guillemin and Kalkman [14 that when $M^{s s}=M^{s}$ the evaluation $\kappa_{M}(\eta)[M / / G]$ of the induced cohomology class $\kappa_{M}(\eta) \in H^{*}(M / / G)$ on the fundamental class of $M / / G$ is equal to a constant multiple, independent of $\eta \in H_{K}^{*}(M)$, of the evaluation on the fundamental class of $M / / T_{c}$ of the cohomology class $\kappa_{M}^{T}\left(\eta \mathcal{D}^{2}\right)$ on $M / / T_{c}$ induced by $\eta \mathcal{D}^{2} \in$ $H_{T}^{*}(M)$. Here $\eta$ and $\mathcal{D}$ are regarded as elements of $H_{T}^{*}(M)$ via the natural maps $H_{K}^{*}(M) \rightarrow H_{T}^{*}(M)$ and $H_{T}^{*} \rightarrow H_{T}^{*}(M)$. Indeed it follows from (1.4) that if $n_{0}^{T}$ is the order of the stabilizer in $T$ of a generic point of $\mu_{T}^{-1}(0)$ then

$$
\kappa_{M}(\eta)[M / / G]=\frac{n_{0}(-1)^{n_{+}}}{n_{0}^{T}|W|} \kappa_{M}^{T}\left(\eta \mathcal{D}^{2}\right)\left[M / / T_{c}\right]
$$

when $M^{s s}=M^{s}$ (although we have to be careful how we interpret the right hand side of this equation if semistability is not the same as stability for the torus action), and Martin [30] has given a direct proof of this which also shows that $\kappa_{M}^{T}(\eta \mathcal{D})\left[M / / T_{c}\right]$ is, up to a sign $(-1)^{n_{+}}$which depends on a choice of orientation, the evaluation on the fundamental class of $\mu^{-1}(0) / T$ of the cohomology class induced by $\eta \in H_{T}^{*}(M)$. If $M^{s s} \neq M^{s}$ then we can apply (1.6) to the blow-up $\tilde{M}$ of $M$ obtained in the construction of the partial desingularization $\tilde{M} / / G$ of $M / / G$.

Next we use the second stage of the approach to nonabelian localization due to Guillemin-Kalkman and to Martin, which involves studying the symplectic quotients $\mu_{T}^{-1}(\xi) / T$ as $\xi$ varies in $\mathbf{t}^{*}$. Since $T$ is abelian, $\mu_{t}-\xi: M \rightarrow \mathbf{t}^{*}$ is a moment map for the action of $T$ on $M$ and $\mu_{T}^{-1}(\xi) / T$ is a symplectic quotient which, when $\xi$ is rational, can be identified with the geometric invariant theoretic quotient $M / / T_{c}$ of $M$ by the complex torus $T_{c}$ with respect to a modified linearization.

Recall that the image $\mu_{T}(M)$ of the moment map $\mu_{T}$ for the action of the maximal torus $T$ of $K$ is a convex polytope; indeed $\mu_{T}$ is constant on the connected components $F \in \mathcal{F}$ of the fixed point set $M^{T}$ for the action of $T$ on $M$, and so $\mu_{T}\left(M^{T}\right)$ is a finite set, whose convex hull is $\mu_{T}(M)$ [1, 15]. The convex polytope $\mu_{T}(M)$ is a union of subpolytopes, each of which is the convex hull of a subset of the finite set $\mu_{T}\left(M^{T}\right)$ and contains no points of $\mu_{T}\left(M^{T}\right)$ in its interior. The interior of each such subpolytope consists of regular values of $\mu_{T}$. The boundaries (or 'walls') between subpolytopes consist of the critical values of the moment map $\mu_{T}$, and are the images under $\mu_{T}$ of the fixed point sets of one-parameter subgroups of $T$. The evaluation on the fundamental class of $\mu_{T}^{-1}(\xi) / T$ of the cohomology class induced by any $\eta \in H_{T}^{*}(M)$ is unchanged as $\xi \in \mathbf{t}^{*}$ varies within a connected component of the set of regular values of $\mu_{T}$, and in 14 formulas are obtained for the change in this evaluation as $\xi$ crosses a wall. Applying these formulas to 
the blow-up $\tilde{M}$ of $M$, we find that it is possible to choose $\xi \in \mathbf{t}^{*}$ which is a regular value of both $\mu_{T}$ and $\tilde{\mu}_{T}$, with the following two properties. Firstly the difference between

$$
\kappa_{\tilde{M}}^{T}\left(\eta \mathcal{D}^{2}\right)\left[\tilde{M} / / T_{c}\right]
$$

and the evaluation on the fundamental class of $\tilde{\mu}_{T}^{-1}(\xi) / T=\tilde{M} / / \xi T_{c}$ of the cohomology class induced by $\eta \mathcal{D}^{2} \in H_{T}^{*}(M)$ can be calculated in terms of data determined purely by the construction of $\tilde{M}^{s s}$ from $M^{s s}$, which is canonical and explicit, rather than the construction of $\tilde{M}$ from $M$, which is neither canonical nor explicit. Secondly this evaluation on $\left[\tilde{M} / / \xi T_{c}\right]$ is equal to the evaluation on the fundamental class of $\mu_{T}^{-1}(\xi) / T$ of the cohomology class induced by $\eta \mathcal{D}^{2}$, which can be calculated by using the residue formula (1.4) applied to the action of $T$ on $M$ with the moment map $\mu_{T}-\xi$. This combined with (1.6) enables us to calculate pairings in the cohomology of the partial desingularization $\tilde{M} / / G$ of $M / / G$.

In order to understand pairings in $I H^{*}(M / / G)$ of intersection cohomology classes on the singular quotient $M / / G$ we make use of the work of the second author in 23]. First note that the composition $\kappa_{M}: H_{K}^{*}(M) \rightarrow I H^{*}(M / / G)$ at (1.5) factors as the composition of the restriction map $H_{K}^{*}(M) \rightarrow H_{K}^{*}\left(M^{s s}\right)$ and a surjection

$$
\kappa_{M}^{s s}: H_{K}^{*}\left(M^{s s}\right) \rightarrow I H^{*}(M / / G) .
$$

In 23] it is shown that if the action of $G$ on $M$ is weakly balanced (see Definition 5.3 below), then there is a naturally defined subset $V_{M}$ of $H_{K}^{*}\left(M^{s s}\right)$ such that $\kappa_{M}^{s s}: H_{K}^{*}\left(M^{s s}\right) \rightarrow I H^{*}(M / / G)$ restricts to an isomorphism

$$
\kappa_{M}^{s s}: V_{M} \rightarrow I H^{*}(M / / G) .
$$

In [23] it is also shown that the intersection pairing of two elements $\kappa_{M}(\alpha)$ and $\kappa_{M}(\beta)$ of complementary degrees in $I H^{*}(M / / G)$ is equal to the evaluation of the image in $H^{*}(\tilde{M} / / G)$ of the product $\alpha \beta \in H_{K}^{*}(M)$ on the fundamental class $[\tilde{M} / / G]$, provided that $\left.\alpha\right|_{M^{s s}}$ and $\left.\beta\right|_{M^{s s}}$ lie in $V_{M}$. In Section 8 below we shall show that if $\left.\alpha\right|_{M^{s s}}$ and $\left.\beta\right|_{M^{s s}}$ lie in $V_{M}$ and are of complementary degrees with respect to $M / / G$, then the intersection pairing of $\kappa_{M}(\alpha)$ and $\kappa_{M}(\beta)$ in $I H^{*}(M / / G)$ is given, just as at (1.4), by

$$
\left\langle\kappa_{M}(\alpha), \kappa_{M}(\beta)\right\rangle_{I H^{*}(M / / G)}=\frac{n_{0}(-1)^{s+n_{+}}}{|W| \operatorname{vol}(T)} \operatorname{res}\left(\mathcal{D}(X)^{2} \sum_{F \in \mathcal{F}_{+}} \int_{F} \frac{i_{F}^{*}\left(\alpha \beta e^{\bar{\omega}}\right)(X)}{e_{F}(X)}[d X]\right),
$$

provided that the multivariable residue res and the subset $\mathcal{F}_{+}$of $\mathcal{F}$ are interpreted correctly. In the case when $T$ is one-dimensional, as before we can take $\mathcal{F}_{+}$to be the set of those $F \in \mathcal{F}$ such that $\mu_{T}(F)$ is positive. The difference now is that there may be some $F \in \mathcal{F}$ with $\mu_{T}(F)=0$, which cannot happen when semistability coincides with stability; this suggests that we need to be careful to decide whether $\mathcal{F}_{+}$consists of those $F \in \mathcal{F}$ for which $\mu_{T}(F)$ is non-negative, or just those for which $\mu_{T}(F)$ is strictly positive. However it 
turns out that when $\alpha$ and $\beta$ lie in $V_{M}$ then

$$
\operatorname{res}\left(\mathcal{D}(X)^{2} \sum_{F \in \mathcal{F}, \mu_{T}(F)=0} \int_{F} \frac{i_{F}^{*}\left(\alpha \beta e^{\bar{\omega}}\right)(X)}{e_{F}(X)}[d X]\right)=0,
$$

so in fact it does not matter which definition of $\mathcal{F}_{+}$we choose here, and the situation is similar when $\operatorname{dim} T>1$.

We can also consider Witten's integral $I^{\epsilon}\left(\eta e^{i \bar{\omega}}\right)$. When 0 is a regular value of the moment map $\mu$ (or equivalently when semistability is the same as stability) then, as we have seen, $I^{\epsilon}\left(\eta e^{i \bar{\omega}}\right)$ can be expressed as a sum of terms which tend to 0 exponentially fast with $\epsilon$, together with

$$
\kappa_{M}(\eta) e^{i \omega_{0}+\epsilon \Theta}[M / / G]
$$

which is a polynomial in $\epsilon$ and can be expressed using the residue formula (1.4) as a sum over the components $F \in \mathcal{F}$ of $M^{T}$. When 0 is not a regular value of $\mu$ we can still write Witten's integral $I^{\epsilon}\left(\eta e^{i \bar{\omega}}\right)$ as a sum of exponentially small terms together with a sum over the components $F \in \mathcal{F}$ of $M^{T}$ (see Section 9 below). The terms in this sum indexed by $F \in \mathcal{F}$ such that $\mu_{T}(F)$ does not lie on a wall through 0 are exactly as they would be in the case when 0 is a regular value of $\mu$, i.e. the residue of

$$
\frac{n_{0}(-1)^{s+n_{+}}}{|W| \operatorname{vol}(T)}\left(\mathcal{D}(X)^{2} e^{-\epsilon<X, X>/ 2} \int_{F} \frac{i_{F}^{*}\left(\eta e^{i \bar{\omega}}\right)(X)}{e_{F}(X)}[d X]\right) .
$$

In particular these terms are polynomials in $\epsilon$. However the terms indexed by $F \in \mathcal{F}$ such that $\mu_{T}(F)$ does lie on a wall through 0 are in general only polynomials in $\epsilon^{1 / 2}$, and it is unclear whether the sum can be interpreted in terms of intersection pairings when 0 is not a regular value of $\mu$.

The construction of the partial desingularization $\tilde{M} / / G$ can also be carried out in the symplectic category, using symplectic blow-ups, to give a partial desingularization of the symplectic reduction of any Hamiltonian $K$ action on a compact symplectic manifold [32, 40]. Because symplectic blowups depend on a number of choices the partial desingularizations obtained will not be unique up to symplectomorphism, but they will be determined up to symplectic homotopy, and in particular up to diffeomorphism. The analysis of Witten's integral $I^{\epsilon}\left(\eta \epsilon^{i \bar{\omega}}\right)$ and the formulas for pairings in $H^{*}(\tilde{M} / / G)$ and $I H^{*}(M / / G)$ are also valid for singular symplectic reductions.

In 39] Witten studied the moduli spaces $\mathcal{M}(n, d)$ of holomorphic bundles of rank $n$ and degree $d$ over a fixed compact Riemann surface $\Sigma$ as symplectic reductions of infinite dimensional affine spaces by infinite dimensional Lie groups. When the rank $n$ and degree $d$ of the bundles are coprime (i.e. when semistability is the same as stability and the moduli space $\mathcal{M}(n, d)$ is nonsingular) then using physical methods Witten obtained formulas (later proved using different methods in [22]) for intersection pairings on these moduli spaces from asymptotic expansions of the integrals $I^{\epsilon}\left(\eta e^{i \bar{\omega}}\right)$ as $\epsilon$ tends to 0 . 
He also gave formulas for the asymptotic expansions of the integrals in the simplest case when semistability differs from stability, namely the case of bundles of rank two and even degree, and he noted that powers of $\epsilon^{1 / 2}$ appeared. In a forthcoming article [19] we will use the finite dimensional methods of 22 together with the results of this paper to rederive Witten's calculations for $\mathcal{M}(2,0)$ and give formulas for intersection pairings in $\operatorname{IH}^{*}(\mathcal{M}(n, d))$ and on the partial resolution of singularities of the moduli space $\mathcal{M}(n, d)$, in the general case for $n \geq 2$ when $n$ and $d$ may have common factors so that $\mathcal{M}(n, d)$ may have singularities.

The layout of this paper is as follows. In Section 2 we recall briefly the relationship between geometric invariant theory (GIT) and the moment map in symplectic geometry, and the use of equivariant cohomology to study the cohomology of GIT quotients. In Section 3 we review Witten's principle of nonabelian localization and the residue formula of [20] in the case when 0 is a regular value of the moment map. In Section 4 we recall the construction of the partial desingularization $\tilde{M} / / G$. In Section 5 we review intersection cohomology and the work of the second author in [23, and in Section 6 we study intersection pairings in $I H^{*}(M / / G)$ via the isomorphism $\kappa_{M}^{s s}: V_{M} \rightarrow I H^{*}(M / / G)$ from [23]. In Section 7 we give formulas for pairings in the intersection cohomology $I H^{*}(M / / G)$ of the singular quotient, and in Section 8 we calculate pairings in the cohomology $H^{*}(\tilde{M} / / G)$ of the partial desingularization. Finally in Section 9 we study Witten's integral.

\section{The moment map and cohomology of quo- tients}

In this section we shall recall briefly the relationship between geometric invariant theory and the moment map in symplectic geometry (see e.g. 34 or 25. for more details), and the use of equivariant cohomology to study the cohomology of geometric invariant theoretic quotients.

Let $M$ be a nonsingular connected complex projective variety, and let $G$ be a connected complex reductive group acting on $M$. In order to define the geometric invariant theoretic quotient $M / / G$ we need a linearization of the action of $G$ on $M$; i.e. we need a lift of the action to a linear action on a line bundle $L$ over $M$, which is usually assumed to be ample. We shall suppose for simplicity that $M$ is embedded in a complex projective space $\mathbb{P}_{n}$ and that $L$ is the hyperplane line bundle on $M$; then we need the action of $G$ to be given by a representation $\rho: G \rightarrow G L(n+1)$. The quotient $M / / G$ is the projective variety defined by the finitely generated graded subalgebra of $\bigoplus_{k \geq 0} H^{0}\left(M, L^{\otimes k}\right)$ consisting of all elements invariant under the action of $G$.

There is a surjective $G$-invariant morphism $\tau: M^{s s} \rightarrow M / / G$ from an open $G$-invariant subset $M^{s s}$ of $M$ (whose elements are called semistable 
points of $M)$ to $M / / G$; in fact $x \in M$ is semistable if and only if there is a $G$-invariant section of $L^{\otimes k}$ for some $k$ which does not vanish at $x$. If $x$ and $y$ are semistable points of $M$ then $\tau(x)=\tau(y)$ if and only if the closures of the orbits $G x$ and $G y$ meet in $M^{s s}$. There is an open $G$-invariant subset $M^{s}$ of $M^{s s}$ (whose elements are called stable ${ }^{2}$ points of $M$ ) such that every fibre of $\tau$ which meets $M^{s}$ is a single $G$-orbit of dimension equal to the dimension of $G$. We shall assume that $M^{s}$ is nonempty; then the image of $M^{s}$ in $M / / G$ is open and dense and can be identified naturally with the ordinary topological quotient $M^{s} / G$.

We shall call elements of $M^{s s}-M^{s}$ strictly semistable, and write $M^{s s s}=$ $M^{s s}-M^{s}$.

The subsets $M^{s s}$ and $M^{s}$ of $M$ are characterized by the following properties (see Chapter 2 of [34] or 35]).

Proposition 1 (i) A point $x \in M$ is semistable (respectively stable) for the action of $G$ on $M$ if and only if for every $g \in G$ the point $g x$ is semistable (respectively stable) for the action of a fixed maximal (complex) torus of $G$.

(ii) A point $x \in M$ with homogeneous coordinates $\left(x_{0}, \ldots, x_{n}\right)$ in some coordinate system on $\mathbb{P}_{n}$ is semistable (respectively stable) for the action of a maximal (complex) torus of $G$ acting diagonally on $\mathbb{P}_{n}$ with weights $\alpha_{0}, \ldots, \alpha_{n}$ if and only if the convex hull

$$
\operatorname{Conv}\left\{\alpha_{i}: x_{i} \neq 0\right\}
$$

contains 0 (respectively contains 0 in its interior).

Now let $K$ be a maximal compact subgroup of $G$; then $G$ is the complexification of $K$. By choosing coordinates on $\mathbb{P}_{n}$ appropriately we may assume that $K$ acts unitarily. Then $K$ preserves the Kähler structure on $M$ given by the restriction of the Fubini-Study metric on $\mathbb{P}_{n}$. The Kähler form $\omega$ makes $M$ into a symplectic manifold on which $K$ acts. Associated to this action there is a moment map $\mu: M \rightarrow \mathbf{k}^{*}$, where $\mathbf{k}$ is the Lie algebra of $K$, given in homogeneous coordinates $x=\left(x_{0}, \ldots, x_{n}\right)$ by the formula

$$
\mu(x) . a=\left(2 \pi i\|x\|^{2}\right)^{-1} x \rho_{*}(a) \bar{x}^{t}
$$

for $a \in \mathbf{k}$. Here the element $\rho_{*}(a)$ of the Lie algebra of the unitary group $U(n+1)$ is thought of as an $n+1$ by $n+1$ skew-hermitian matrix. Recall that the defining property of a moment map $\mu: M \rightarrow \mathbf{k}^{*}$ is that

$$
d \mu(x)(\xi) \cdot a=\omega_{x}(\xi, \tilde{a}(x))
$$

for all $x \in M, \xi \in T_{x} M$ and $a \in \mathbf{k}$, where $\tilde{a}$ is the vector field on $M$ induced by $a$. We also require that $\mu$ carries the given $K$-action on $M$ to the coadjoint action on the dual of its Lie algebra.

\footnotetext{
${ }^{2}$ This is now the usual terminology and notation. However in 34 the terminology "properly stable" and notation $M_{(0)}^{s}$ are used instead.
} 
When a compact group $K$ acts on a symplectic manifold $M$ and $\mu$ : $M \rightarrow \mathbf{k}^{*}$ is a moment map for the action, the symplectic form on $M$ induces a symplectic form on the quotient $\mu^{-1}(0) / K$ (away from its singularities, at least), which is the Marsden-Weinstein reduction or symplectic quotient of $M$ by the action of $K$. In our situation $\mu^{-1}(0) / K$ can be identified with the geometric invariant theoretic quotient $M / / G$. A more precise statement is the following (see [34] Theorem 8.2 and Remark 8.3 or [25] 6.2, 8.10, 7.2 and $7.5)$.

Proposition 2 (i) $x \in M^{s s}$ if and only if $\mu^{-1}(0)$ meets the closure of the orbit $G x$ in $M$.

(ii) $x \in M^{s}$ if and only if $\mu^{-1}(0)$ meets the orbit $G x$ at a point whose stabilizer in $K$ is finite.

(iii) The inclusion of $\mu^{-1}(0)$ in $M^{\text {ss }}$ induces a homeomorphism $\mu^{-1}(0) / K \rightarrow$ $M / / G$.

If the Lie algebra $\mathbf{k}$ is given a fixed $K$-invariant inner product then we can consider the function $\|\mu\|^{2}$ as a Morse function on $M$ (although it is not a Morse function in the classical sense; see [25]). It induces a Morse stratification of $M$, in which the stratum containing any $x \in M$ is determined by the limit set of its path of steepest descent for $\|\mu\|^{2}$ (with respect to the Kähler metric). This stratification can also be defined purely algebraically, and has the following properties (see 25] 5.4 and Chapters 12 and 13).

Proposition 3 (i) Each stratum is a G-invariant locally closed nonsingular subvariety of $M$.

(ii) $M^{s s}$ coincides with the unique open stratum.

(iii) The stratification is equivariantly perfect (that is, its equivariant Morse inequalities are all equalities) over the complex numbers, and in particular the restriction map

$$
H_{K}^{*}(M) \rightarrow H_{K}^{*}\left(M^{s s}\right) \cong H_{K}^{*}\left(\mu^{-1}(0)\right)
$$

is surjective.

Here the $K$-equivariant cohomology of any topological space $Y$ on which $K$ acts is

$$
H_{K}^{*}(Y)=H^{*}\left(Y \times_{K} E K\right)
$$

where $E K \rightarrow B K$ is the universal $K$-bundle. (Recall that all cohomology groups have complex coefficients throughout this paper). Note that $K$ is homotopically equivalent to its complexification $G$, so $G$-equivariant cohomology is naturally isomorphic to $K$-equivariant cohomology; we shall work with the latter in this paper. 
If $M$ is a manifold, the $K$-equivariant cohomology of $M$ can be identified with the cohomology of a chain complex $\Omega_{K}^{*}(M)$ whose elements are $K$ equivariant polynomial functions on the Lie algebra $\mathbf{k}$ of $K$ with values in the de Rham complex $\Omega^{*}(M)$ of differential forms on $M$ (see for example Chapter 7 of [5]). We shall call elements of $\Omega_{K}^{*}(M)$ equivariant differential forms on $M$. The differential $D$ on this complex is defined by ${ }^{3}$

$$
(D \eta)(X)=d(\eta(X))-\iota_{X \#}(\eta(X))
$$

where $X^{\#}$ is the vector field on $M$ generated by the action of $X$ (see Chapter 7 of $[\underline{5})$. We can write $\Omega_{K}^{*}(M)=\left(S\left(\mathbf{k}^{*}\right) \otimes \Omega^{*}(M)\right)^{K}$ where $S\left(\mathbf{k}^{*}\right)$ denotes the algebra of polynomial functions on the Lie algebra $\mathbf{k}$ of $K$. An element $\eta \in \Omega_{K}^{*}(M)$ may be thought of as a $K$-equivariant polynomial function from $\mathbf{k}$ to $\Omega^{*}(M)$, or alternatively as a family of differential forms on $M$ parametrized by $X \in \mathbf{k}$. The standard definition of degree is used on $\Omega^{*}(M)$ and degree two is assigned to elements of $\mathbf{k}^{*}$.

In fact, as a vector space though not in general as a ring, when $M$ is a compact symplectic manifold with a Hamiltonian action of $K$ then $H_{K}^{*}(M)$ is isomorphic to $H^{*}(M) \otimes H_{K}^{*}$ where $H_{K}^{*}=\Omega_{K}^{*}(\mathrm{pt})=S\left(\mathbf{k}^{*}\right)^{K}$ is the equivariant cohomology of a point (see 25] Proposition 5.8).

It follows directly from the defining property of a moment map that if $\mu$ is regarded in the obvious way as a linear map from $\mathbf{k}$ to the space $\Omega^{0}(Y)$ of smooth complex-valued functions on $Y$, then $\bar{\omega} \in \Omega_{K}^{2}(M)$ defined by

$$
\bar{\omega}(X)=\omega+\mu(X)
$$

satisfies $D \bar{\omega}=0$ and therefore defines an extension of the cohomology class of $\omega$ in $H^{2}(M)$ to an equivariant cohomology class in $H_{K}^{2}(M)$.

If every semistable point of $M$ is stable then by Proposition $2 K$ acts on $\mu^{-1}(0)$ with only finite stabilizers. Because of the defining property (2.1) of a moment map, this implies that 0 is a regular value of $\mu$ and hence that $\mu^{-1}(0)$ is a submanifold of $M$. Since the cohomology with complex coefficients of a classifying space of a finite group is always trivial, it also implies that the obvious map

$$
\mu^{-1}(0) \times_{K} E K \rightarrow \mu^{-1}(0) / K
$$

induces an isomorphism

$$
H^{*}\left(\mu^{-1}(0) / K\right) \cong H_{K}^{*}\left(\mu^{-1}(0)\right)
$$

and hence $H^{*}(M / / G) \cong H_{K}^{*}\left(M^{s s}\right)$. Composing this with the surjection of Proposition 3(iii), we find that if $M^{s s}=M^{s}$ then there is a natural surjective ring homomorphism from $H_{K}^{*}(M)$ to $H^{*}(M / / G)$.

\footnotetext{
${ }^{3}$ This definition of the equivariant cohomology differential differs by a factor of $i$ from that used in [39] but is consistent with that used in [20, 22].
} 
Example 4 Consider the action of $G=S L(2)$ and its maximal compact subgroup $K=S U(2)$ on $\mathbb{P}_{n}$ identified with the space of unordered sequences of $n$ points in $\mathbb{P}_{1}$ (that is, with the projectivized symmetric product $\left.\mathbb{P}\left(S^{n}\left(\mathbb{C}^{2}\right)\right)\right)$. The diagonal subgroup $\mathbb{C}^{*}$ is a maximal torus of $G$ and acts with weights $n, n-2, n-4, \ldots, 2-n,-n$ on $S^{n}\left(\mathbb{C}^{2}\right)=\mathbb{C}^{n+1}$. An element $\left[a_{0}, \ldots, a_{n}\right]$ of $\mathbb{P}_{n}$ corresponds to the $n$ roots in $\mathbb{P}_{1}$ of the polynomial with coefficients $a_{0}, \ldots, a_{n}$; it is semistable (respectively stable) for the action of $G$ if and only if at most $n / 2$ (respectively strictly fewer than $n / 2$ ) of these roots coincide anywhere on $\mathbb{P}_{1}$. The induced stratification of $M$ has strata $S_{0}=M^{s s}$ and $S_{j}$ for $n / 2<j \leq n$. If $n / 2<j \leq n$ then the elements of $S_{j}$ correspond to sequences of $n$ points on $\mathbb{P}_{1}$ such that exactly $j$ of these points coincide somewhere on $\mathbb{P}_{1}$, and $S_{j}$ retracts equivariantly onto the subset of $M$ where $j$ points coincide somewhere on $\mathbb{P}_{1}$ and the remaining $n-j$ points coincide somewhere else on $\mathbb{P}_{1}$. This subset is a single $G$-orbit with stabilizer $\mathbb{C}^{*}$, so that

$$
H_{K}^{*}\left(S_{j}\right) \cong H^{*}\left(B \mathbb{C}^{*}\right) \cong H^{*}\left(B S^{1}\right),
$$

and the fact that the stratification is equivariantly perfect tells us that

$$
\operatorname{dim} H_{K}^{q}\left(M^{s s}\right)=\operatorname{dim} H_{K}^{q}(M)-\sum_{n / 2<j \leq n} \operatorname{dim} H_{K}^{q-\operatorname{dim}_{\mathbb{R}}\left(S_{j}\right)}\left(S_{j}\right) .
$$

The same is true when $M=\left(\mathbb{P}_{1}\right)^{n}$, except that then $S_{j}$ has $\left(\begin{array}{c}n \\ j\end{array}\right)$ components, each of which retracts onto a single $G$-orbit and has equivariant cohomology isomorphic to $H^{*}\left(B S^{1}\right)$ (see 25] Section 9 for more details).

Example 5 (Example 6.3 in $[23]$.) Consider the $\mathbb{C}^{*}$-action on $\mathbb{P}^{7}$ by a representation with weights $+1,0,-1$ with multiplicity $3,2,3$ respectively. Then

$$
H_{S^{1}}^{*}\left(\mathbb{P}^{7}\right)=\mathbb{C}[\xi, \rho] /<\xi^{2}(\xi-\rho)^{3}(\xi+\rho)^{3}>
$$

is the quotient of the polynomial ring $\mathbb{C}[\xi, \rho]$ where $\xi$ is a generator in $H^{2}\left(\mathbb{P}^{7}\right)$ and $\rho$ is a generator in $H^{2}\left(B S^{1}\right)$, by the ideal generated by $\xi^{2}(\xi-\rho)^{3}(\xi+$ $\rho)^{3}$. There are two unstable strata whose equivariant cohomology classes are $\xi^{2}(\xi-\rho)^{3}$ and $\xi^{2}(\xi+\rho)^{3}$. Since the Morse stratification with respect to the norm square of the moment map is equivariantly perfect,

$$
H_{S^{1}}^{*}\left(\left(\mathbb{P}^{7}\right)^{s s}\right)=\mathbb{C}[\xi, \rho] /<\xi^{2}(\xi-\rho)^{3}, \xi^{2}(\xi+\rho)^{3}>
$$

A Gröbner basis for the relation ideal is

$$
\left\{\xi^{5}+3 \xi^{3} \rho^{2}, \xi^{4} \rho+\frac{1}{3} \xi^{2} \rho^{3}, \xi^{3} \rho^{3}, \xi^{2} \rho^{5}\right\}
$$

where $\xi>\rho$. Hence as a vector space,

$$
H_{S^{1}}^{*}\left(\left(\mathbb{P}^{7}\right)^{s s}\right) \cong \mathbb{C}\left\{\xi^{i} \rho^{j}: i=0,1, j \geq 0\right\} \oplus \mathbb{C}\left\{\xi^{i} \rho^{j}: 2 i+j<9, i \geq 2, j \geq 0\right\} .
$$




\section{Residue formulas and nonabelian localiza- tion}

The map $\Omega_{K}^{*}(M) \rightarrow \Omega_{K}^{*}(\mathrm{pt})=S\left(\mathbf{k}^{*}\right)^{K}$ given by integration over $M$ passes to $H_{K}^{*}(M)$. Thus for any $D$-closed element $\eta \in \Omega_{K}^{*}(M)$ representing a cohomology class $[\eta]$, there is a corresponding element $\int_{M} \eta \in \Omega_{K}^{*}(\mathrm{pt})$ which depends only on $[\eta]$. The same is true for any $D$-closed formal series $\eta=\sum_{j} \eta_{j}$ of elements $\eta_{j}$ in $\Omega_{K}^{j}(M)$ : we shall in particular consider terms of the form

$$
\eta(X) e^{\bar{\omega}(X)}
$$

where $\eta \in \Omega_{K}^{*}(M)$ and

$$
\bar{\omega}(X)=\omega+\mu(X) \in \Omega_{K}^{2}(M) .
$$

If $X$ lies in $\mathbf{t}$, the Lie algebra of a maximal torus $T$ of $K$, then there is a formula for $\int_{M} \eta(X)$ (the abelian localization formula [3, 5, 6, 7]) which depends only on the fixed point set of $T$ in $M$. It tells us that

$$
\int_{M} \eta(X)=\sum_{F \in \mathcal{F}} \int \frac{i_{F}^{*} \eta(X)}{e_{F}(X)}
$$

where $\mathcal{F}$ indexes the components $F$ of the fixed point set of $T$ in $M$, the inclusion of $F$ in $M$ is denoted by $i_{F}$ and $e_{F} \in H_{T}^{*}(M)$ is the equivariant Euler class of the normal bundle to $F$ in $M$. In particular, applying (3.1) with $\eta$ replaced by the formal equivariant cohomology class $\eta e^{\bar{\omega}}$ we have

$$
\int_{M} \eta(X) e^{\bar{\omega}(X)}=\sum_{F \in \mathcal{F}} h_{F}^{\eta}(X)
$$

where

$$
h_{F}^{\eta}(X)=e^{\mu(F)(X)} \int_{F} \frac{i_{F}^{*} \eta(X) e^{\omega}}{e_{F}(X)} .
$$

Note that the moment map $\mu$ takes a constant value $\mu(F)=\mu_{T}(F) \in \mathbf{t}^{*}$ on each $F \in \mathcal{F}$, and that the integral in (3.3) is a rational function of $X$.

The main result (the residue formula, Theorem 8.1) of [20] gives a formula for the evaluation on the fundamental class $[M / / G] \in H_{*}(M / / G)$, or equivalently (if we represent cohomology classes by differential forms) the integral over $M / / G$, of the image $\kappa_{M}(\eta) e^{\omega_{0}}$ in $H^{*}(M / / G)$ of any formal equivariant cohomology class on $M$ of the type $\eta e^{\bar{\omega}}$ where $\eta \in H_{K}^{*}(M)$.

Theorem 6 (i) (Residue formula, [20] Theorem 8.1) Let $\eta \in H_{K}^{*}(M)$ induce $\kappa_{M}(\eta) \in H^{*}(M / / G)$. Then

$$
\kappa_{M}(\eta) e^{\omega_{0}}[M / / G]=n_{0} C_{K} \operatorname{res}\left(\mathcal{D}^{2}(X) \sum_{F \in \mathcal{F}} h_{F}^{\eta}(X)[d X]\right),
$$


where the constant ${ }^{4} C_{K}$ is defined by

$$
C_{K}=\frac{(-1)^{s+n_{+}}}{|W| \operatorname{vol}(T)}
$$

and $n_{0}$ is the order of the stabilizer in $K$ of a generic point of $\mu^{-1}(0)$.

(ii) (Reduction to an integral over $\mu^{-1}(0) / T,[29]$ )

$$
\kappa_{M}(\eta) e^{\omega_{0}}[M / / G]=\frac{n_{0}}{n_{0}^{T}|W|} \int_{\mu^{-1}(0) / T} \kappa_{M}^{T}\left(\mathcal{D}(X) \eta e^{\bar{\omega}}\right),
$$

where $n_{0}^{T}$ is the order of the stabilizer in $T$ of a generic point of $\mu^{-1}(0)$.

In these formulas $|W|$ is the order of the Weyl group $W$ of $K$, while $s=\operatorname{dim} K$ and $l=\operatorname{dim} T$, and $n_{+}=(s-l) / 2$ is the number of positive roots. The measure $[d X]$ on $\mathbf{t}$ and volume vol $(T)$ of $T$ are obtained from the restriction of a fixed invariant inner product on $\mathbf{k}$, which is used to identify $\mathbf{k}^{*}$ with $\mathbf{k}$ throughout. Also, $\mathcal{F}$ denotes the set of components of the fixed point set of $T$, and if $F$ is one of these components then the meromorphic function $h_{F}^{\eta}$ on $\mathbf{t} \otimes \mathbb{C}$ is defined by (3.3). The polynomial $\mathcal{D}: \mathbf{t} \rightarrow \mathbb{R}$ is defined by

$$
\mathcal{D}(X)=\prod_{\gamma>0} \gamma(X)
$$

where $\gamma$ runs over the positive roots of $K$. Note that it would perhaps be more natural to combine $(-1)^{n_{+}}$from the constant $C_{K}$ with $\mathcal{D}^{2}(X)$ and replace them by the product

$$
\prod_{\gamma} \gamma(X)
$$

of all the positive and negative roots of $K$.

Let $\mu_{T}: M \rightarrow \mathbf{t}^{*}$ be the composition of the moment map $\mu: M \rightarrow \mathbf{k}^{*}$ with the restriction map from $\mathbf{k}^{*}$ to $\mathbf{t}^{*}$; then $\mu_{T}$ is a moment map for the action of $T$ on $M$. In particular $\mu_{T}$ is constant on any connected component $F$ of the fixed point set $M^{T}$ for the action of $T$ on $M$.

The multivariable residue res which appears in the formula (3.4) above can be thought of as a linear map defined on a certain class of meromorphic differential forms on $\mathbf{t} \otimes \mathbb{C}$, but in order to apply it to the individual terms in the residue formula it is necessary to make some choices which do not affect the residue of the whole sum. Once the choices have been made, many of the terms in the sum contribute zero and the formula can be rewritten as a sum over a subset $\mathcal{F}_{+}$of the set $\mathcal{F}$ of components of the fixed point set $M^{T}$, consisting of those $F \in \mathcal{F}$ on which the constant value taken by $\mu_{T}$ lies

\footnotetext{
${ }^{4}$ In this paper we are adopting the conventions of 22 on the equivariant differential and on the normalization of weights (see Footnote 9 on page 125 of 22 for the effect of different conventions on the constant $C_{K}$ ).
} 
in a certain cone with its vertex at 0 . When the rank of $K$ is one and $\mathbf{t}$ is identified with $\mathbb{R}$, we can take

$$
\mathcal{F}_{+}=\left\{F \in \mathcal{F}: \mu_{T}(F)>0\right\}
$$

When $K=U(1)$, then the residue formula becomes

$$
\kappa_{M}(\eta) e^{\omega_{0}}[M / / G]=-n_{0} \operatorname{res}_{X=0}\left(\sum_{F \in \mathcal{F}_{+}} h_{F}^{\eta}(X) d X\right)
$$

where $\operatorname{res}_{X=0}$ denotes the coefficient of $1 / X$ when $X \in \mathbb{R}$ has been identified with $2 \pi i X \in \mathbf{k}$. When $K=S U(2)$ we have

$$
\kappa_{M}(\eta) e^{\omega_{0}}[M / / G]=\frac{n_{0}}{2} \operatorname{res}_{X=0}\left((2 X)^{2} \sum_{F \in \mathcal{F}_{+}} h_{F}^{\eta}(X)\right)
$$

when $X \in \mathbb{R}$ has been identified with $\operatorname{diag}(2 \pi i,-2 \pi i) X \in \mathbf{t}$.

Example 7 When $K=S U(2)$ with maximal torus $T=S^{1}$ acts on $M=$ $\left(\mathbb{P}_{1}\right)^{n}$, the equivariant cohomology $H_{T}^{*}(M)$ of $M$ with respect to $T$ is generated by $n$ elements $\xi_{1}, \ldots, \xi_{n}$ of degree two which are lifts of the standard generators of $H^{*}(M)$, together with another generator $\zeta$ of degree two coming from $H_{T}^{*}$, subject to the relations

$$
\left(\xi_{1}\right)^{2}=\ldots=\left(\xi_{n}\right)^{2}=\zeta^{2}
$$

$H_{K}^{*}$ is generated by $\xi_{1}, \ldots, \xi_{n}$ and $\zeta^{2}$ subject to the same relations. We assume that $n$ is odd, so that 0 is a regular value of $\mu$, or equivalently semistability coincides with stability for the action of the complexification $G=S L(2)$ of $K$ (cf. Example 2.4). When $\mathbb{P}_{1}$ is identified with the unit sphere $S^{2}$ in $\mathbb{R}^{3}$ and the dual of the Lie algebra of $S U(2)$ is identified suitably with $\mathbb{R}^{3}$ the moment map is given by

$$
\mu\left(x_{1}, \ldots, x_{n}\right)=x_{1}+\ldots+x_{n} .
$$

The fixed point sets of the action of the standard maximal torus $T$ of $K$ on $M$ are the $n$-tuples $\left(x_{1}, \ldots, x_{n}\right) \in\left(\mathbb{P}_{1}\right)^{n}$ such that each $x_{j}$ is either 0 or $\infty$. If we index these by sequences $\left(\delta_{1}, \ldots, \delta_{n}\right)$ where $\delta_{j}=1$ if $x_{j}=0$ and $\delta_{j}=-1$ if $x_{j}=\infty$, then (3.7) gives us the formula

$$
\begin{gathered}
\kappa_{M}\left(q\left(\xi_{1}, \ldots, \xi_{n}, \zeta^{2}\right)\right) e^{\omega_{0}}[M / / G]= \\
\operatorname{res}_{X=0}\left(4 X^{2} \sum_{\left(\delta_{1}, \ldots, \delta_{n}\right) \in\{1,-1\}^{n}, \delta_{1}+\ldots+\delta_{n}>0} \frac{q\left(\delta_{1} X, \ldots, \delta_{n} X, X^{2}\right) e^{\left(\delta_{1}+\ldots+\delta_{n}\right) X}}{\left(\prod_{j} \delta_{j}\right) X^{n}}\right)
\end{gathered}
$$

for any polynomial $q\left(\xi_{1}, \ldots, \xi_{n}, \zeta^{2}\right)$ in the generators $\xi_{1}, \ldots, \xi_{n}$ and $\zeta^{2}$ for $H_{K}^{*}(M)$ (see Section 9 of [20]). 
Remark 8 If we suppose that the degree of $\eta$ is equal to the real dimension of $M / / G$ then of course we have $\kappa_{M}(\eta) e^{\omega_{0}}[M / / G]=\kappa_{M}(\eta)[M / / G]$. In order that the multivariable residue res which appears in the general version (3.4) of the residue formula should be defined, we still need to include the terms $e^{\mu(F)(X)}$ and $e^{\omega}$ coming from $e^{\bar{\omega}}$ in the right hand side of (3.4). However we can omit them from the right hand side of (3.6) and (3.7) if we wish; they have done their job in reducing the sum over $F \in \mathcal{F}$ to a sum over $F \in \mathcal{F}_{+}$.

Note that (see 20, 2.7) the reciprocal of the $T$-equivariant Euler class $e_{F}(X)$ can be expressed in the form

$$
\frac{1}{e_{F}(X)}=\prod_{j=1}^{N_{F}} \frac{1}{c_{1}\left(\nu_{F, j}\right)+\beta_{F, j}(X)}=\prod_{j=1}^{N_{F}} \sum_{r_{j} \geq 0} \frac{\left(-c_{1}\left(\nu_{F, j}\right)\right)^{r_{j}}}{\beta_{F, j}(X)^{r_{j}+1}}
$$

where $\beta_{F, 1}, \ldots, \beta_{F, N_{F}}$ are the weights of the action of $T$ on the normal bundle to $F$ in $M$, and $c_{1}\left(\nu_{F, 1}\right), \ldots, c_{1}\left(\nu_{F, N_{F}}\right) \in H^{2}(F)$ are nilpotent. Thus the terms $\mathcal{D}^{2}(X) h_{F}^{\eta}(X)$ appearing in the residue formula can all be expressed as finite sums of functions of the form

$$
h(X)=\frac{q(X) e^{\lambda(X)}}{\prod_{j=1}^{N} \beta_{j}(X)},
$$

where $q(X)$ is a polynomial in $X \in \mathbf{t}$ and $\lambda(X)$ and $\beta_{1}(X), \ldots, \beta_{N}(X)$ are linear functions of $X$. It is shown in Proposition 3.2 of [21] and Proposition 8.11 of [20] that the multivariable residue of $h(X)[d X]$ when $h(X)$ has this form is determined completely by a few elementary properties. Alternatively res can be expressed in terms of iterated one-dimensional residues using Proposition 3.4 of [21].

Remark 9 Note that the multivariable residue defined and used in [20, 21 is very slightly different from the one used here and in [22, because in [20, 21] the residue formula is applied to formal equivariant cohomology classes of the form $\eta e^{i \bar{\omega}}$ instead of $\eta e^{\bar{\omega}}$. The factors of $i$ were omitted in [22] because they are essentially irrelevant to the residue formula, although they appear naturally in Witten's integral $I^{\epsilon}\left(\eta e^{i \bar{\omega}}\right)$. In [20, 21, and also Section 9 of this paper, functions of the form $q(X) e^{i \lambda(X)} / \prod_{j=1}^{N} \beta_{j}(X)$ replace the functions of the form $q(X) e^{\lambda(X)} / \prod_{j=1}^{N} \beta_{j}(X)$ studied here and in [22]. To obtain the elementary properties which uniquely determine the multivariable residue used here and in 22], one simply omits all the occurrences of $i$ in [21] Proposition 3.2 (see also Section 9 below).

\section{Partial resolution of singularities}

In this section we shall describe the construction of the partial resolution of singularities $\tilde{M} / / G \rightarrow M / / G$ (for more details see [26]). 
As before let $M$ be a nonsingular complex projective variety embedded in a projective space $\mathbb{P}_{n}$ and let $G$ be a connected complex reductive group acting on $M$ via a representation $\rho: G \rightarrow G L(n+1)$. Let $V$ be any nonsingular $G$-invariant closed subvariety of $M$ and let $\pi: \hat{M} \rightarrow M$ be the blowup of $M$ along $V$. The linear action of $G$ on the hyperplane line bundle $L$ over $M$ lifts to a linear action on the line bundle over $\hat{M}$ which is the pullback of $L^{\otimes k}$ tensored with $\mathcal{O}(-E)$, where $E$ is the exceptional divisor and $k$ is a fixed positive integer. When $k$ is large the line bundle $\pi^{*} L^{\otimes k} \otimes \mathcal{O}(-E)$ is ample on $\hat{M}$, so there is an embedding of $\hat{M}$ in a projective space such that a positive tensor power of this line bundle is isomorphic to the restriction of the hyperplane line bundle on the projective space. It is proved in Section 3 of [26] that when $k$ is large enough this linear action satisfies the following properties:

(i) If $y$ is semistable in $\hat{M}$ then $\pi(y)$ is semistable in $M$.

(ii) If $\pi(y)$ is stable in $M$ then $y$ is stable in $\hat{M}$.

The rough idea of the proof is to use Proposition 2.1 in conjunction with the facts that if $k>0$ then the stability and semistability with respect to $L^{\otimes k}$ of a point of $M$ is independent of $k$, and that when $k$ is large the weights of the action on $H^{0}\left(\hat{M}, \pi^{*} L^{\otimes k} \otimes \mathcal{O}(-E)\right)$ of a maximal torus of $G$ can be thought of as small perturbations of the weights of its action on $H^{0}\left(\hat{M}, \pi^{*} L^{\otimes k}\right)$. A similar argument shows that if $k$ is sufficiently large then the sets $\hat{M}^{s}$ and $\hat{M}^{s s}$ of stable and semistable points of $\hat{M}$ with respect to this linearization are independent of $k$.

Remark 10 Note that the induced symplectic form $\hat{\omega}$ on $\hat{M}$ and moment map $\hat{\mu}: \hat{M} \rightarrow \mathbf{k}^{*}$ are of the form

$$
\hat{\omega}=k \pi^{*} \omega+\Omega \text { and } \hat{\mu}=k \mu \circ \pi+\nu
$$

where $\Omega$ and $\nu$ are independent of $k$. Thus if $k \gg 0$ then after scaling by $1 / k$ (which does not change the quotient) the symplectic form and moment map for $\hat{M}$ are small perturbations $\pi^{*} \omega+(1 / k) \Omega$ and $\mu \circ \pi+(1 / k) \nu$ of the pullbacks to $\hat{M}$ of the symplectic form and moment map for $M$.

Now $M$ has semistable points which are not stable if and only if there exists a nontrivial connected reductive subgroup of $G$ which fixes some semistable point. If so, let $r>0$ be the maximal dimension of the reductive subgroups of $G$ fixing semistable points of $M$, and let $\mathcal{R}(r)$ be a set of representatives of conjugacy classes in $G$ of all connected reductive subgroups $R$ of dimension $r$ such that

$$
Z_{R}^{s s}=\left\{x \in M^{s s}: R \text { fixes } x\right\}
$$

is nonempty. Then

$$
\bigcup_{R \in \mathcal{R}(r)} G Z_{R}^{s s}
$$


is a disjoint union of nonsingular closed subvarieties of $M^{s s}$, and

$$
G Z_{R}^{s s} \cong G \times_{N^{R}} Z_{R}^{s s}
$$

where $N^{R}$ is the normalizer of $R$ in $G$.

By Hironaka's theorem [18] we can resolve the singularities of the closure of $\bigcup_{R \in \mathcal{R}(r)} G Z_{R}^{s s}$ in $M$ by performing a sequence of blow-ups along nonsingular $G$-invariant closed subvarieties of $M-M^{s s}$. We then blow up along the proper transform of the closure of $\bigcup_{R \in \mathcal{R}(r)} G Z_{R}^{s s}$ to get a nonsingular projective variety $\hat{M}_{1}$. The linear action of $G$ on $M$ lifts to an action on this blow-up $\hat{M}_{1}$ which can be linearized using suitable ample line bundles as above, and it is shown in [26] that the set $\hat{M}_{1}^{s s}$ of semistable points of $\hat{M}_{1}$ with respect to any of these suitable linearizations of the lifted action is the complement in the inverse image of $M^{s s}$ of the proper transform of the subset

$$
\phi^{-1}\left(\phi\left(\bigcup_{R \in \mathcal{R}(r)} G Z_{R}^{s s}\right)\right)
$$

of $M^{s s}$, where $\phi: M^{s s} \rightarrow M / / G$ is the canonical map. Moreover no point of $\hat{M}_{1}^{s s}$ is fixed by a reductive subgroup of $G$ of dimension at least $r$, and a point in $\hat{M}_{1}^{s s}$ is fixed by a reductive subgroup $R$ of $G$ of dimension less than $r$ if and only if it belongs to the proper transform of the subvariety $Z_{R}^{s s}$ of $M^{s s}$.

The same procedure is now applied in [26] to $\hat{M}_{1}$ to obtain $\hat{M}_{2}$ such that no reductive subgroup of $G$ of dimension at least $r-1$ fixes a point of $\hat{M}_{2}^{s s}$. After repeating enough times we obtain $\tilde{M}$ satisfying $\tilde{M}^{s s}=\tilde{M}^{s}$, and then the induced map $\tilde{M} / / G \rightarrow M / / G$ is a partial resolution of singularities.

Remark 11 If we are only interested in $\tilde{M}^{s s}$ and the partial resolution $\tilde{M} / / G$ of $M / / G$, rather than in $\tilde{M}$ itself, then there is no need in this procedure to resolve the singularities of the closure of $\bigcup_{R \in \mathcal{R}(r)} G Z_{R}^{s s}$ in $M$. Instead we can simply blow $M^{s s}$ up along $\bigcup_{R \in \mathcal{R}(r)} G Z_{R}^{s s}$ (or equivalently along each $G Z_{R}^{s s}$ in turn) and let $\hat{M}_{1}^{s s}$ be the set of semistable points in the result.

Example 12 Let $G=S L(2)$ act on $M=\left(\mathbb{P}_{1}\right)^{n}$ and suppose that $n$ is even, so that semistability and stability do not coincide (see Example 2.4). The semistable elements of $M$ which are fixed by nontrivial connected reductive subgroups of $G$ are those of the form $\left(x_{1}, \ldots, x_{n}\right)$ such that there exist distinct $p$ and $q$ in $\mathbb{P}_{1}$ with exactly half of the points $x_{1}, \ldots, x_{n}$ equal to $p$ and the rest equal to $q$. They form

$$
\frac{n !}{2((n / 2) !)^{2}}
$$

$G$-orbits and their stabilizers are all conjugate to the maximal torus $T_{c}=\mathbb{C}^{*}$ of $G$. We obtain the partial desingularization $\tilde{M} / / G$ by blowing up $M / / G$ at the points corresponding to these orbits, or equivalently by blowing up 
$M^{s s}$ along these orbits, removing the unstable points from the blowup (these form the proper transform of the set of $\left(x_{1}, \ldots, x_{n}\right) \in M^{s s}$ such that exactly half of the points $x_{1}, \ldots, x_{n}$ coincide somewhere on $\mathbb{P}_{1}$ ) and finally quotienting by $G$.

\section{Intersection homology and a splitting of the surjection $\kappa_{M}^{s s}: H_{K}^{*}\left(M^{s s}\right) \rightarrow I H^{*}(M / / G)$}

In this section, we shall recall the splitting constructed in [23] (see also 24]) of the surjection $\kappa_{M}^{s s}: H_{K}^{*}\left(M^{s s}\right) \rightarrow I H^{*}(M / / G)$ defined at (1.7).

Let $W$ be a (singular) complex projective variety. Then it has a filtration $W=W_{m} \supseteq W_{m-1} \supseteq \cdots \supseteq W_{0}$ by closed subvarieties which defines a Whitney stratification of $W$ with nonsingular strata $W_{j}-W_{j-1}$ of complex dimension $j$, and its intersection cohomology $I H^{*}(W)$ with complex coefficients and with respect to the middle perversity can be defined as follows [11, 12. Let $I C^{2 m-i}(W)$ be the group of chains $\sigma$ of dimension $i$ in $W$ such that

$$
\begin{aligned}
\operatorname{dim}_{\mathbb{R}}\left(|\sigma| \cap W_{m-k}\right) & \leq i-k-1 \\
\operatorname{dim}_{\mathbb{R}}\left(|\partial \sigma| \cap W_{m-k}\right) & \leq i-k-2
\end{aligned}
$$

Then $I C^{*}(W)$ is a chain complex whose cohomology is the intersection cohomology $I H^{*}(W)$ of $W$. It does not depend on the choice of the stratification and it is a homeomorphism invariant [11, 12. It coincides with ordinary cohomology for nonsingular varieties, and also for orbifolds since we are using complex coefficients. If $\sigma \in I C^{*}(W)$ then neither $\sigma$ nor $\partial \sigma$ can be contained in $W_{m-1}$, so if $L$ is any local coefficient system on the nonsingular open subset $W-W_{m-1}$ of $W$ then we can define a chain complex $I C^{*}(W, L)$ of intersection chains in $W$ with coefficients in $L$, and thus define the intersection homology $I H^{*}(W, L)$ of $W$ with coefficients in $L$.

Any two intersection cohomology classes of complementary degrees in $W$ can be represented by cycles in $W$ which intersect transversely and only on the nonsingular part of $W$ at a finite number of points. If we count these intersection points with appropriate signs we obtain a nondegenerate pairing on $I H^{*}(W)$ which is called the intersection pairing. Thus $I H^{*}(W)$ satisfies Poincaré duality. It also satisfies the properties of the cohomology of

smooth compact Kähler manifolds known as the Kähler package, including the existence of a Hodge structure and the hard Lefschetz property.

One of the most useful tools for working with intersection cohomology is the decomposition theorem of Beilinson, Bernstein, Deligne and Gabber [4] which tells us that if $f: A \rightarrow B$ is a projective map of complex varieties then there exist closed subvarieties $B_{\alpha}$ of $B$ and local systems $L_{\alpha}$ on open dense 
subsets of $B_{\alpha}$ such that

$$
I H^{i}(A) \cong \bigoplus_{\alpha} I H^{i-l_{\alpha}}\left(B_{\alpha}, L_{\alpha}\right)
$$

for suitable integers $l_{\alpha}$. If $f$ is birational then there is some $\alpha$ such that $B_{\alpha}=B$ and $L_{\alpha}=\mathbb{C}$ and $l_{\alpha}=0$, so that $I H^{*}(B)$ appears as a direct summand of $I H^{*}(A)$ in this decomposition. In particular, the intersection cohomology $I H^{*}(M / / G)$ of the GIT quotient $M / / G$ can be regarded as a direct summand of the ordinary cohomology $H^{*}(\tilde{M} / / G)$ of its partial desingularization $\tilde{M} / / G$, so we get a surjection

$$
H^{*}(\tilde{M} / / G) \rightarrow I H^{*}(M / / G) .
$$

The decomposition (5.3) is unfortunately not in general canonical, but in our situation the hard Lefschetz theorem can be used to make a canonical choice of decomposition and hence a canonical choice of surjection $H^{*}(\tilde{M} / / G) \rightarrow$ $I H^{*}(M / / G)$ (see [27].)

Our goal is to understand the intersection cohomology of the singular quotient $M / / G$ in terms of the equivariant cohomology of $M$. A procedure for computing the intersection cohomology Betti numbers $\operatorname{dim} I H^{j}(M / / G)$ is given in [27], and it was generalized to symplectic quotients in [40]. One can compute the equivariant Poincaré series

$$
P_{t}^{K}\left(M^{s s}\right)=\sum_{j \geq 0} t^{j} \operatorname{dim} H_{K}^{j}\left(M^{s s}\right)
$$

of $M^{s s}$ by equivariant Morse theory applied to the function $\|\mu\|^{2}$ as in 25 ] (cf. Proposition 2.3 above), and keep track of the equivariant Poincaré series while blowing up until one reaches the partial desingularization, and then switch to intersection cohomology while blowing down until one comes back to $M / / G$ [24, 27, 40]. The switch is possible since $\tilde{M}^{s s}=\tilde{M}^{s}$, so that $H_{K}^{*}\left(\tilde{M}^{s s}\right)$ is isomorphic to $H^{*}(\tilde{M} / / G)=I H^{*}(\tilde{M} / / G)$.

Example 13 When $K=S U(2)$ acts on $M=\mathbb{P}_{n}$ we have

$$
P_{t}^{K}(M)=\left(1+t^{2}+t^{4}+\ldots+t^{2 n}\right)\left(1-t^{4}\right)^{-1}
$$

and

$$
P_{t}^{K}\left(M^{s s}\right)=P_{t}^{K}(M)-\sum_{n / 2<j \leq n} t^{2(j-1)}\left(1-t^{2}\right)^{-1}
$$

(see Example 2.4). If $n$ is odd so that semistability and stability coincide then this is a polynomial of degree $2(n-3)$ in $t$ whose coefficients are the (intersection) Betti numbers of $M / / G$. If $n$ is even, then to obtain the partial desingularization $\tilde{M} / / G$ one must blow up $M^{s s}$ along the orbit of the element $[0, \ldots, 0,1,0, \ldots, 0] \in \mathbb{P}_{n}$ corresponding to the polynomial whose roots in $\mathbb{P}_{1}$ are 
0 and $\infty$, each with multiplicity $n / 2$, and then remove the unstable points from the blowup. This gives us

$$
\begin{gathered}
P_{t}(\tilde{M} / / G)=P_{t}^{K}\left(\tilde{M}^{s s}\right) \\
=P_{t}^{K}\left(M^{s s}\right)+\left(t^{2}+t^{4}+\ldots+t^{2(n-3)}\right)\left(1-t^{4}\right)^{-1}-t^{n-2}\left(1+t^{2}+\ldots+t^{n-4}\right)\left(1-t^{2}\right)^{-1} \\
=1+2 t^{2}+3 t^{4}+4 t^{6}+\ldots+\left(\frac{n}{2}-2\right) t^{n-6}+\left(\frac{n}{2}-1\right) t^{n-4}+ \\
+\left(\frac{n}{2}-1\right) t^{n-2}+\left(\frac{n}{2}-2\right) t^{n}+\ldots+3 t^{2 n-10}+2 t^{2 n-8}+t^{2 n-6} .
\end{gathered}
$$

Finally we study the kernel of the surjection from $H^{*}(\tilde{M} / / G)$ to $I H^{*}(M / / G)$ to obtain the intersection Poincaré polynomial of $M / / G$ as

$$
\begin{gathered}
I P_{t}(M / / G)=P_{t}(\tilde{M} / / G)-\left(t^{2}+t^{4}+2 t^{6}+2 t^{8}+\ldots+[(n-2) / 4] t^{n-4}+\right. \\
\left.+[(n-2) / 4] t^{n-2}+\ldots+t^{2 n-10}+t^{2 n-8}\right) \\
=1+t^{2}+2 t^{4}+2 t^{6}+\ldots+[n / 4] t^{n-4}+[n / 4] t^{n-2}+\ldots+2 t^{2 n-10}+t^{2 n-8}+t^{2 n-6},
\end{gathered}
$$

where $[a]$ is the integer part of $a$. For more details see [27].

The composition of the maps in the partial desingularization process gives us a map from $\tilde{M}^{s s}$ to $M^{s s}$ and hence a ring homomorphism $H_{K}^{*}\left(M^{s s}\right) \rightarrow$ $H_{K}^{*}\left(\tilde{M}^{s s}\right)$. Via the decomposition theorem the corresponding maps on quotients induce surjections on intersection cohomology whose composition gives us our surjection from $H^{*}(\tilde{M} / / G)=I H^{*}(\tilde{M} / / G)$ to $I H^{*}(M / / G)$. In this way we get

$$
\kappa_{M}^{s s}: H_{K}^{*}\left(M^{s s}\right) \rightarrow H_{K}^{*}\left(\tilde{M}^{s s}\right) \cong I H^{*}(\tilde{M} / / G) \rightarrow I H^{*}(M / / G) .
$$

This map $\kappa_{M}^{s s}: H_{K}^{*}\left(M^{s s}\right) \rightarrow I H^{*}(M / / G)$ is surjective; the proof of this in [27] is flawed but an alternative proof is given in [41].

In order to get useful information about the intersection cohomology $I H^{*}(M / / G)$, a splitting of the map $\kappa_{M}^{s s}: H_{K}^{*}\left(M^{s s}\right) \rightarrow I H^{*}(M / / G)$ was constructed in 23, 24] under the assumption that the linear action of $G$ on $M$ is weakly balanced in the sense defined below.

Definition 14 Suppose a nontrivial connected reductive group $R$ acts on a vector space $A$ linearly. Let $\mathcal{B}$ be the set of the closest points from the origin to the convex hulls of some weights of the action. For each $\beta \in \mathcal{B}$, denote by $n(\beta)$ the number of weights $\alpha$ such that $\alpha \cdot \beta<\beta \cdot \beta$. The action is said to be weakly linearly balanced if $2 n(\beta)-2 \operatorname{dim}_{\mathbb{C}} R / B \operatorname{Stab} \beta>\operatorname{dim}_{\mathbb{C}} A-\operatorname{dim}_{\mathbb{C}} R$ for every $\beta \in \mathcal{B}$ where $B$ is a Borel subgroup of $R$.

Let $\mathcal{R}$ be a set of representatives of the conjugacy classes in $G$ of subgroups which appear as identity components of stabilizers of points $x \in M^{s s}$ such that $G x$ is closed in $M^{s s}$. Such subgroups are always connected reductive subgroups of $G$ (see e.g. [28]). 
Definition 15 Let $G$ be a connected reductive group acting linearly on a connected nonsingular quasi-projective variety $M$. The $G$-action is said to be weakly balanced if for each $R \in \mathcal{R}$ the linear action of $R$ on the normal space $\mathcal{N}_{x}$ at any $x \in Z_{R}^{s}$ to $G Z_{R}^{s s}$ is weakly linearly balanced and so is the action of $\left(R \cap N^{g R^{\prime} g^{-1}}\right) / g R^{\prime} g^{-1}$ on the $g R^{\prime} g^{-1}$-fixed linear subspace $\mathcal{N}_{x}^{g R^{\prime} g^{-1}}=$ $Z_{g R^{\prime} g^{-1}}^{s s} \cap \mathcal{N}_{x}$ for each $R^{\prime} \in \mathcal{R}$ satisfying $g R^{\prime} g^{-1} \subseteq R$.

For example, a $\mathbb{C}^{*}$ action on $\mathbb{P}^{n}$ is weakly balanced if and only if the number (counting multiplicities) of positive weights is same as the number of negative weights. The actions described in Examples 2.4 and 2.5 of $S L(2)$ and its maximal torus $\mathbb{C}^{*}$ on $\mathbb{P}_{n}$ and $\left(\mathbb{P}_{1}\right)^{n}$ are weakly balanced. More examples are provided by the (compactified) moduli spaces of holomorphic vector bundles of any rank and degree over a fixed Riemann surface (see Example 3.5 of [23]).

For $R \in \mathcal{R}$, we consider the natural map

$$
G \times_{N^{R}} Z_{R}^{s s} \rightarrow G Z_{R}^{s s}
$$

where $N^{R}$ is the normalizer of $R$ in $G$, and the corresponding map

$$
\left.H_{K}^{*}\left(G Z_{R}^{s s}\right) \rightarrow H_{K}^{*}\left(G \times_{N^{R}} Z_{R}^{s s}\right) \cong H_{N^{R}}^{*}\left(Z_{R}^{s s}\right) \cong\left[H_{N_{0}^{R} / R}^{*}\left(Z_{R}^{s s}\right) \otimes H_{R}^{*}\right)\right]^{\pi_{0} N^{R}}
$$

where the subscript 0 means the identity component. For any $\zeta \in H_{K}^{*}\left(M^{s s}\right)$ we let $\left.\zeta\right|_{G \times{ }_{N} R} Z_{R}^{s s}$ denote the image of $\zeta$ under the composition of the above map and the restriction map $H_{K}^{*}\left(M^{s s}\right) \rightarrow H_{K}^{*}\left(G Z_{R}^{s s}\right)$. Then the main result of [23] is the following splitting of the map $\kappa_{M}^{s s}: H_{K}^{*}\left(M^{s s}\right) \rightarrow I H^{*}(M / / G)$ obtained by "truncating along each stratum" when the action is weakly balanced.

Theorem 16 [23] Let

$V_{M}=\left\{\zeta \in H_{K}^{*}\left(M^{s s}\right):\left.\zeta\right|_{G \times_{N} R} Z_{R}^{s s} \in\left[\oplus_{i<n_{R}} H_{N_{0}^{R} / R}^{*}\left(Z_{R}^{s s}\right) \otimes H_{R}^{i}\right]^{\pi_{0} N^{R}}\right.$ for each $\left.R \in \mathcal{R}\right\}$

where $n_{R}=\operatorname{dim}_{\mathbb{C}} \mathcal{N}_{x}-\operatorname{dim}_{\mathbb{C}} R=\operatorname{dim}_{\mathbb{C}} \mathcal{N}_{x} / / R$ and $\mathcal{N}_{x}$ is the normal space to $G Z_{R}^{s s}$ at any $x \in Z_{R}^{s}$. If the action of $G$ is weakly balanced, then the restriction

$$
\kappa_{M}^{s s}: V_{M} \rightarrow I H^{*}(M / / G)
$$

of the $\operatorname{map}_{M}^{s s}: H_{K}^{*}\left(M^{s s}\right) \rightarrow I H^{*}(M / / G)$ is an isomorphism.

Example 17 We continue Example 5. In the terminology of Theorem 16 we have $n_{R}=5$, and so we have to remove $\mathbb{C}\left\{\xi^{i} \rho^{j}: i=0,1, j \geq 3\right\}$ to get $V_{M}$. Hence,

$$
V=\oplus_{0 \leq i \leq 6} V^{2 i}
$$

where

$$
V^{0}=\mathbb{C}, \quad V^{2}=\mathbb{C}\{\rho, \xi\}, \quad V^{4}=\mathbb{C}\left\{\rho^{2}, \xi \rho, \xi^{2}\right\},
$$




$$
\begin{gathered}
V^{6}=\mathbb{C}\left\{\xi \rho^{2}, \xi^{2} \rho, \xi^{3}\right\}, \quad V^{8}=\mathbb{C}\left\{\xi^{2} \rho^{2}, \xi^{3} \rho, \xi^{4}\right\}, \\
V^{10}=\mathbb{C}\left\{\xi^{2} \rho^{3}, \xi^{3} \rho^{2}\right\}, \quad V^{12}=\mathbb{C}\left\{\xi^{2} \rho^{4}\right\} .
\end{gathered}
$$

Therefore, the intersection Poincaré series for $\mathbb{P}^{7} / / \mathbb{C}^{*}$ is

$$
1+2 t^{2}+3 t^{4}+3 t^{6}+3 t^{6}+3 t^{8}+2 t^{10}+t^{12} .
$$

This formula could also be obtained by the sort of calculation described for $\mathbb{P}_{n} / / S L(2)$ in Example 5.1, but such a calculation is usually lengthier.

Remark 18 The equivariant cohomology of $M$ and of $M^{s s}$ and the intersection cohomology of $M / / G$ carry natural Hodge structures, and the maps we have been considering (in particular $\kappa_{M}$ and $\kappa_{M}^{s s}$ ) respect these Hodge structures. Thus we can use these methods to calculate Hodge numbers as well as Betti numbers. In particular if every cohomology class of $M$ is of Hodge type $(p, p)$ for some $p$ then the same is true for $M / / G$, as can be seen in the last example (cf. 25] Section 14).

\section{Intersection pairings via the splitting $V_{M}$}

In this section, we study the intersection pairing in $I H^{*}(M / / G)$ via $V_{M}$. Throughout this section, we assume that the action of $G$ is weakly balanced and thus we have the isomorphism

$$
\kappa_{M}^{s s}: V_{M} \rightarrow I H^{*}(M / / G) \text {. }
$$

Let $\tau$ be the top degree class in $V_{M}$ corresponding to the fundamental class in $I H^{m}(M / / G)$ where $m$ is the real dimension of the quotient $M / / G$. It comes from a class in the compactly supported cohomology group $H_{c}^{m}\left(M^{s} / G\right)$ which can be represented by a closed differential form with compact support, via the composition

$$
H_{c}^{m}\left(M^{s} / G\right) \cong H^{m}\left(M / / G, M^{s s s} / / G\right) \cong H_{K}^{m}\left(M^{s s}, M^{s s s}\right) \rightarrow H_{K}^{m}\left(M^{s s}\right) .
$$

Then we have the following theorem [23].

Theorem 19 Let $\alpha, \beta$ be two classes of complementary degrees in $V_{M}$ with respect to $m$. Then their product $\alpha \beta$ in $H_{K}^{*}\left(M^{s s}\right)$ is the top degree class $\tau$ in $V_{M}$ multiplied by the scalar

$$
\left\langle\kappa_{M}^{s s}(\alpha), \kappa_{M}^{s s}(\beta)\right\rangle_{I H^{*}(M / / G)}
$$

where $\langle\cdot, \cdot\rangle_{I H^{*}(M / / G)}$ denotes the intersection pairing in $I H^{*}(M / / G)$. 
Example 20 We continue Examples 2.5 and 17. Notice that by the Gröbner basis in Example 2.5, $\xi^{3} \rho^{3}=0, \xi^{4} \rho^{2}=-\frac{1}{3} \xi^{2} \rho^{4}, \xi^{6}=\xi^{2} \rho^{4}$, and $\xi^{5} \rho=0$. Hence, for example, the matrix for the pairing $V^{6} \otimes V^{6} \rightarrow \mathbb{C}$ is up to a constant

$$
\left(\begin{array}{ccc}
1 & 0 & -\frac{1}{3} \\
0 & -\frac{1}{3} & 0 \\
-\frac{1}{3} & 0 & 1
\end{array}\right)
$$

One can similarly compute the pairings for other classes.

Since the map $H_{K}^{*}\left(M^{s s}\right) \rightarrow H_{K}^{*}\left(\tilde{M}^{s s}\right) \cong H^{*}(\tilde{M} / / G)$ induced from the maps in the partial desingularization process is a ring homomorphism, the relation

$$
\alpha \beta=\left\langle\kappa_{M}^{s s}(\alpha), \kappa_{M}^{s s}(\beta)\right\rangle_{I H^{*}(M / / G)} \tau
$$

in Theorem 6.1 is preserved. The intersection pairing $\left\langle\kappa_{M}^{s s}(\alpha), \kappa_{M}^{s s}(\beta)\right\rangle_{I H^{*}(M / / G)}$ therefore equals the evaluation $\kappa_{\tilde{M}}^{s s}(\alpha \beta)[\tilde{M} / / G]$ of the image in $H^{*}(\tilde{M} / / G)$ of the product $\alpha \beta \in H_{K}^{*}\left(M^{s s}\right)$ on the fundamental class $[\tilde{M} / / G]$, because $\kappa_{\tilde{M}}^{s s}(\tau)[\tilde{M} / / G]=1$. Hence, we get the following.

Proposition 21 Let $\alpha, \beta$ be classes of complementary degrees in $V_{M}$. Then

$$
\left\langle\kappa_{M}^{s s}(\alpha), \kappa_{M}^{s s}(\beta)\right\rangle_{I H^{*}(M / / G)}=\kappa_{\tilde{M}}^{s s}(\alpha \beta)[\tilde{M} / / G] .
$$

Remark 22 Via the natural map $H^{*}(M / / G) \rightarrow I H^{*}(M / / G)$ the pairing in $I H^{*}(M / / G)$ determines the intersection pairing in the ordinary cohomology $H^{*}(M / / G)$, which may be degenerate for singular quotients. The quotient map $E K \times_{K} M^{s s} \rightarrow M / / G$ induces a ring homomorphism $H^{*}(M / / G) \rightarrow$ $H_{K}^{*}\left(M^{s s}\right)$ which factors through $V_{M}$, and the composition $H^{*}(M / / G) \rightarrow$ $H_{K}^{*}\left(M^{s s}\right) \rightarrow I H^{*}(M / / G)$ is the natural map $H^{*}(M / / G) \rightarrow I H^{*}(M / / G)$ which preserves the pairing.

\section{Pairings in intersection cohomology}

In this section and the next we consider pairings in $I H^{*}(M / / G)$ and pairings in the cohomology $H^{*}(\tilde{M} / / G)$ of classes in the image of the composition

$$
H_{K}^{*}(M) \rightarrow H_{K}^{*}(\tilde{M}) \rightarrow H^{*}(\tilde{M} / / G)
$$

of the pullback from $M$ to $\tilde{M}$ and the map $\kappa_{\tilde{M}}$. As in previous sections we abuse notation by suppressing the pullback and writing $\kappa_{\tilde{M}}(\alpha)$ for the image of $\alpha \in H_{K}^{*}(M)$. Since these maps are ring homomorphisms, such a pairing is given simply by evaluating the product against the fundamental class; i.e.

$$
\left\langle\kappa_{\tilde{M}}(\alpha), \kappa_{\tilde{M}}(\beta)\right\rangle_{H^{*}(\tilde{M} / / G)}=\kappa_{\tilde{M}}(\alpha \beta)[\tilde{M} / / G] .
$$


Furthermore in Section 6] it was shown that if the $G$ action is weakly balanced and $\left.\alpha\right|_{M^{s s}}$ and $\left.\beta\right|_{M^{s s}}$ lie in the subspace $V_{M}$ of $H_{K}^{*}\left(M^{s s}\right)$ which is isomorphic to $I H^{*}(M / / G)$ then

$$
\left\langle\kappa_{\tilde{M}}(\alpha), \kappa_{\tilde{M}}(\beta)\right\rangle_{I H^{*}(M / / G)}=\kappa_{\tilde{M}}(\alpha \beta)[\tilde{M} / / G] .
$$

In this section we will find a formula for $\kappa_{\tilde{M}}(\alpha \beta)[\tilde{M} / / G]$ in this special case; in the next section we will study evaluations of the form $\kappa_{\tilde{M}}(\alpha \beta)[\tilde{M} / / G]$ for any $\alpha, \beta \in H_{K}^{*}(M)$.

Recall that we are assuming that $M$ is a nonsingular complex projective variety embedded in a complex projective space $\mathbb{P}_{n}$, and that $G$ acts on $M$ via a complex representation $\rho: G \rightarrow G L(n+1)$ of $G$ such that $\rho(K) \subseteq$ $U(n+1)$. This representation $\rho$ gives us a lift of the action of $G$ on $M$ to the hyperplane line bundle over $M$, i.e. a linearization of the action of $G$ on $M$. We can change the linearization without changing the action of $G$ on $M$ by multiplying $\rho$ by any character $\chi: G \rightarrow \mathbb{C}^{*}$ of $G$. If we identify $\chi$ with an element of $\mathbf{k}^{*}$ in the usual way by taking the derivative at the identity of the restriction of $\chi$ to $K$, then this change in linearization corresponds to shifting the moment map $\mu: M \rightarrow \mathbf{k}^{*}$ by the central element $\chi$ of $\mathbf{k}^{*}$. The quotient

$$
M / / \chi G
$$

of $M$ by $G$ with respect to this shifted linearization can be identified topologically with the quotient of the Zariski open subset

$$
M_{\chi}^{s s}=\{m \in M \mid \chi \in \mu(\overline{G \cdot m})\}
$$

of $M$ by the equivalence relation $\sim$ such that $x \sim y$ if and only if

$$
\overline{G \cdot x} \cap \overline{G \cdot y} \cap M_{\chi}^{s s} \neq \emptyset \text {. }
$$

Just as we have a homeomorphism $M / / G \cong \mu^{-1}(0) / K$, so we have homeomorphisms

$$
M / /{ }_{\chi} G \cong \mu^{-1}(\chi) / K
$$

for any such $\chi$. Moreover we can generalize the construction of $M_{\chi}^{s s}$ and $M / / \chi G \cong \mu^{-1}(\chi) / K$ to any central element $\chi$ of $\mathbf{k}^{*}$. In particular for any $\xi \in \mathbf{t}^{*}$ we can define

$$
M_{\xi, T}^{s s}=\left\{m \in M \mid \xi \in \mu_{T}\left(\overline{T_{c} \cdot m}\right)\right\}
$$

and its quotient $M / /{ }_{\xi} T_{c}=M_{\xi, T}^{s s} / \sim$ which is homeomorphic to $\mu_{T}^{-1}(\xi) / T$. When $\xi$ is a regular value of $\mu_{T}$ then just as at (1.1) we get a map

$$
H_{T_{c}}^{*}(M) \rightarrow H_{T_{c}}^{*}\left(M_{\xi, T}^{s s}\right) \cong H^{*}\left(M / /{ }_{\xi} T_{c}\right)
$$

which we shall denote by $\kappa_{M, \xi}^{T}$.

By the convexity theorem of Atiyah [1] and Guillemin and Sternberg [17] the image $\mu_{T}(M)$ of the moment map $\mu_{T}$ is a convex polytope in $\mathbf{t}^{*}$ and the 
dense set of regular values is the disjoint union of finitely many open convex subpolytopes

$$
\Delta_{1} \cup \ldots \cup \Delta_{r}
$$

In fact $\mu_{T}(M)$ is the convex hull in $\mathbf{t}^{*}$ of the finite set $\left\{\mu_{T}(F): F \in \mathcal{F}\right\}$, and it is divided by walls of codimension one into subpolytopes which are convex hulls of subsets of $\left\{\mu_{T}(F): F \in \mathcal{F}\right\}$ and whose interiors consist of regular values of $\mu_{T}$. Here $\mathcal{F}$ is the set of components of the fixed point set of the action of $T$ on $M$.

Suppose $\xi \in \Delta_{i}$ and $\zeta \in \overline{\Delta_{i}}$. Then $M_{\xi}^{s s} \subseteq M_{\zeta}^{s s}$ and this inclusion induces a birational map

$$
\mu_{T}^{-1}(\xi) / T \cong M_{\xi}^{s s} / / T_{c} \rightarrow M_{\zeta}^{s s} / / T_{c} \cong \mu_{T}^{-1}(\zeta) / T .
$$

Of course if $\zeta \in \Delta_{i}$ then this map is an isomorphism ${ }^{5}$, but this is not true in general when $\zeta$ lies in the boundary of $\Delta_{i}$.

Proposition 23 Suppose the action of $G$ on $M$ is weakly balanced. For $\alpha, \beta \in H_{K}^{*}(M)$ such that $\left.\alpha\right|_{M^{s s}},\left.\beta\right|_{M^{s s}} \in V_{M}$ we have

$$
\kappa_{\tilde{M}}(\alpha \beta)[\tilde{M} / / G]=\frac{n_{0}(-1)^{n_{+}}}{n_{0}^{T}|W|} \kappa_{M, \xi}^{T}\left(\alpha \beta \mathcal{D}^{2}\right)\left[M / /{ }_{\xi} T_{c}\right]
$$

for any $\xi \in \Delta_{i}$ with $0 \in \overline{\Delta_{i}}$.

Proof: Recall from Theorem [19] and Proposition 21] that if $\alpha$ and $\beta$ are of complementary degree then

$$
\left.\alpha \beta\right|_{M^{s s}}=\kappa_{\tilde{M}}(\alpha \beta)[\tilde{M} / / G] \tau
$$

where $\tau \in H_{K}^{\operatorname{dim} M / / G}\left(M^{s s}\right)$ is the class in $V_{M}$ corresponding to the dual in $I H^{\operatorname{dim} M / / G}(M / / G)$ of the class of a point in $I H_{0}(M / / G)$.

Let $U$ be open in the subset $M^{s} \subseteq M^{s s}$ of stable points in $M$ for the $G$ action (and recall that $U$ is then contained in the stable part of $M$ with respect to the $T_{c}$ action with linearization induced from that of $G$ ). Applying to compactly supported cohomology on $U$ the arguments used by Martin in [29, 30] (see also [22] Section 3) to prove (1.6), we find that $\tau \mathcal{D}^{2} \in H_{T_{c}}^{*}\left(M_{0, T}^{s s}\right)$ is the image under the composition

$$
H_{c}^{\operatorname{dim} M / / T_{c}}\left(U / T_{c}\right) \cong H_{T_{c}, c}^{\operatorname{dim} M / / T_{c}}(U) \rightarrow H_{T_{c}}^{\operatorname{dim} M / / T_{c}}\left(M_{0, T}^{s s}\right)
$$

\footnotetext{
${ }^{5}$ Each face of $\Delta_{i}$ is similarly divided into subpolytopes, and if $\xi$ and $\zeta$ both lie in the interior of the same subpolytope in a face of $\Delta_{i}$ then

$$
\mu_{T}^{-1}(\xi) / T \cong M_{\xi}^{s s} / / T_{c}=M_{\zeta}^{s s} / / T_{c} \cong \mu_{T}^{-1}(\zeta) / T
$$
}


of the unique generator $\gamma$ of $H_{c}^{\operatorname{dim} M / / T_{c}}\left(U / T_{c}\right)$ with the normalization

$$
\gamma\left[U / T_{c}\right]=\frac{n_{0}^{T}|W|}{n_{0}(-1)^{n_{+}}} .
$$

Let us now take $U=M_{\xi, T}^{s s} \cap M^{s}$. There is then a commutative diagram

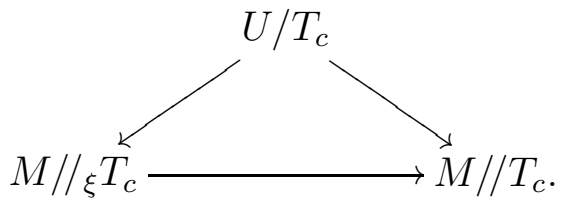

By equation (7.2) we have

$$
\left.\alpha \beta \mathcal{D}^{2}\right|_{M^{s s}}=\left.\kappa_{\tilde{M}}(\alpha \beta)[\tilde{M} / / G] \tau \mathcal{D}^{2}\right|_{M^{s s}} .
$$

So, using the above diagram and description of $\tau \mathcal{D}^{2}$, we see that

$$
\begin{aligned}
\frac{n_{0}(-1)^{n_{+}}}{n_{0}^{T}|W|} \kappa_{M, p}^{T}\left(\alpha \beta \mathcal{D}^{2}\right)\left[M / / \xi T_{c}\right] & =\kappa_{\tilde{M}}(\alpha \beta)[\tilde{M} / / G]\left(\frac{n_{0}(-1)^{n_{+}} \gamma}{n_{0}^{T}|W|}\left[U / T_{c}\right]\right) \\
& =\kappa_{\tilde{M}}(\alpha \beta)[\tilde{M} / / G]
\end{aligned}
$$

as required.

Remark 24 This result can be viewed as an extension of the well known fact that for $\xi, \zeta \in \Delta_{i}$ and any $\eta \in H_{T_{c}}^{*}(M)$ we have

$$
\kappa_{M, \xi}^{T}(\eta)\left[M / / \xi T_{c}\right]=\kappa_{M, \zeta}^{T}(\eta)\left[M / / \zeta T_{c}\right] .
$$

Provided the action is weakly balanced with respect to $\zeta^{6}$ and we restrict $\eta$ to be a scalar multiple of $\tau \mathcal{D}^{2}$ then the same formula is valid when $\zeta \in \overline{\Delta_{i}} \backslash \Delta_{i}$.

Since $\xi$ is a regular value of $\mu_{T}$ we can evaluate

$$
\kappa_{M, \xi}^{T}\left(\alpha \beta \mathcal{D}^{2}\right)\left[M / / \xi T_{c}\right]
$$

using a residue formula as at (1.4) which involves a sum over the set $\mathcal{F}$ of fixed point components of the $T$ action on $M$. Together with the results of Section [6 this allows us, when the $G$ action is weakly balanced, to write down residue formulae for computing pairings in the intersection cohomology $I H^{*}(M / / G)$, as follows.

Theorem 25 Suppose that the $G$ action on $M$ is weakly balanced and that $\alpha, \beta \in H_{K}^{*}(M)$ have degrees whose sum is the real dimension of $M / / G$. Suppose also that $\left.\alpha\right|_{M^{s s}}$ and $\left.\beta\right|_{M^{s s}}$ lie in the subspace $V_{M}$ of $H_{K}^{*}\left(M^{s s}\right)$ which is isomorphic to $I H^{*}(M / / G)$. Then

$$
\left\langle\kappa_{M}(\alpha), \kappa_{M}(\beta)\right\rangle_{I H^{*}(M / / G)}=\kappa_{\tilde{M}}(\alpha \beta)[\tilde{M} / / G]
$$

\footnotetext{
${ }^{6}$ By "weakly balanced with respect to $\zeta$ " we mean weakly balanced with respect to the moment map $\mu-\zeta$, in other words we subtract $\zeta$ from all the weights.
} 


$$
=\frac{n_{0}(-1)^{s+n_{+}}}{|W| \operatorname{vol}(T)} \operatorname{res}\left(\mathcal{D}^{2} \sum_{F \in \mathcal{F}} \int_{F} \frac{i_{F}^{*}\left(\alpha \beta e^{(\bar{\omega}-\epsilon)}\right)(X)}{e_{F}}[d X]\right)
$$

for any sufficiently small $\epsilon \in \mathbf{t}^{*}$ which is a regular value of the moment map $\mu_{T}$.

Example 26 Let us consider again the action of $G=S L(2)$ on $M=\mathbb{P}_{n}$ (Example 2.4 and 5.1). The equivariant cohomology $H_{T}^{*}(M)$ of $M$ with respect to the maximal torus $T$ of $K=S U(2)$ is generated by two equivariant cohomology classes $\xi$ and $\zeta$, both of degree two, subject to the relation

$$
(\xi-n \zeta)(\xi-(n-2) \zeta) \ldots(\xi+(n-2) \zeta)(\xi+n \zeta)=0,
$$

where the nonidentity element of the Weyl group $W$ of $K$ sends $\zeta$ to $-\zeta$ and fixes $\xi$. Thus the equivariant cohomology $H_{K}^{*}(M)$ of $M$ with respect to $K$ is generated to $\xi$ and $\zeta^{2}$ subject to the same relation. The fixed point sets for the action of $T$ are the $n+1$ points represented by the weight vectors; the values taken by the moment map on the fixed points are just the weights $-n, 2-n, \ldots, n-2, n$ up to a universal scalar multiple. If $n$ is odd then semistability equals stability, and if $\eta \in H_{K}^{*}(M)$ has degree $2(n-3)=$ $\operatorname{dim}_{\mathbb{R}}(M / / G)$ and is given by a polynomial $q\left(\xi, \zeta^{2}\right)$ in the generators $\xi$ and $\zeta^{2}$, then the residue formulas (1.4) or (3.7) give us

$$
\begin{aligned}
\kappa_{M}(\eta)[M / / G] & =\operatorname{res}_{X=0}\left(4 X^{2} \sum_{j: 0<n-2 j \leq n} \frac{q\left((n-2 j) X, X^{2}\right)}{\prod_{k \neq j}((n-2 k) X-(n-2 j) X)}\right) \\
& =\operatorname{res}_{X=0}\left(\sum_{j: 0<n-2 j \leq n} \frac{q\left((n-2 j) X, X^{2}\right)}{(2 X)^{n-2} \prod_{k \neq j}(k-j)}\right)
\end{aligned}
$$

(cf. Example 3.2). If, on the other hand, $n$ is even then Theorem 7.3 shows us that if $\alpha, \beta \in H_{K}^{*}(M)$ satisfy $\operatorname{deg}(\alpha)+\operatorname{deg}(\beta)=2(n-3)$ and if $\left.\alpha\right|_{M^{s s}}$ and $\left.\beta\right|_{M^{s s}}$ lie in the subspace $V_{M}$ of $H_{K}^{*}\left(M^{s s}\right)$ then

$$
\begin{gathered}
\left\langle\kappa_{M}(\alpha), \kappa_{M}(\beta)\right\rangle_{I H^{*}(M / / G)}=\kappa_{\tilde{M}}(\alpha \beta)[\tilde{M} / / G] \\
=\operatorname{res}_{X=0} \sum_{j: 0<n-2 j \leq n} \frac{q\left((n-2 j) X, X^{2}\right)}{(2 X)^{n-2} \prod_{k \neq j}(k-j)}
\end{gathered}
$$

where $\alpha \beta=q\left(\xi, \zeta^{2}\right)$.

\section{Pairings on the partial desingularization}

In this section we consider pairings $\kappa_{\tilde{M}}(\alpha \beta)[\tilde{M} / / G]$ in the cohomology $H^{*}(\tilde{M} / / G)$ of classes $\kappa_{\tilde{M}}(\alpha)$ and $\kappa_{\tilde{M}}(\beta)$ in the image of the composition

$$
H_{K}^{*}(M) \rightarrow H_{K}^{*}(\tilde{M}) \rightarrow H^{*}(\tilde{M} / / G)
$$


of the pullback from $M$ to $\tilde{M}$ and the map $\kappa_{\tilde{M}}$.

First note that applying (1.6) to $\tilde{M}$ we have

$$
\kappa_{\tilde{M}}(\alpha \beta)[\tilde{M} / / G]=\frac{n_{0}(-1)^{n_{+}}}{n_{0}^{T}|W|} \kappa_{\tilde{M}}^{T}\left(\alpha \beta \mathcal{D}^{2}\right)\left[\tilde{M} / / T_{c}\right]
$$

Remark 27 As at (1.6) (cf. Theorem 3.1(ii)) we can replace the pairing $\kappa_{\tilde{M}}^{T}\left(\alpha \beta \mathcal{D}^{2}\right)\left[\tilde{M} / / T_{c}\right]$ by the evaluation on $\left[\tilde{\mu}^{-1}(0) / T\right]$ of the cohomology class induced by $(-1)^{n_{+}} \alpha \beta \mathcal{D}$ if we want to make sure that we are only working with the semistable part $\tilde{M}^{s s}$ of $\tilde{M}$. Indeed we can think of $(-1)^{n_{+}} \mathcal{D}$ as representing the Poincaré dual to $\tilde{\mu}^{-1}(0) / T$ in $\tilde{\mu}_{T}^{-1}(0) / T=\tilde{M} / / T_{c}$ or the equivariant Poincaré dual to $\tilde{\mu}^{-1}(0)$ in $\tilde{\mu}_{T}^{-1}(0)$.

Now let $\xi$ be any regular value of the $T$-moment map $\mu_{T}$ for $M$. Let $\tilde{M}_{\xi, T}^{s s}$ be defined as at (17.1); then it follows from Section 3 of [26] that if we choose $k \gg 0$ and if $x \in \tilde{M}$ lies in $\tilde{M}_{\xi, T}^{s s}$ then its image $\pi(x)$ in $M$ lies in $M_{\xi, T}^{s s}$. Thus $\pi: \tilde{M} \rightarrow M$ induces a birational morphism

$$
\pi_{\xi}: \tilde{M} / /{ }_{\xi} T_{c} \rightarrow M / /{ }_{\xi} T_{c}
$$

and we have $\left(\pi_{\xi}\right)_{*}\left[\tilde{M} / /{ }_{\xi} T_{c}\right]=\left[M / / \xi T_{c}\right]$. Since $\pi_{\xi}: H^{*}\left(M / / \xi T_{c}\right) \rightarrow H^{*}\left(\tilde{M} / / \xi T_{c}\right)$ fits into the commutative diagram

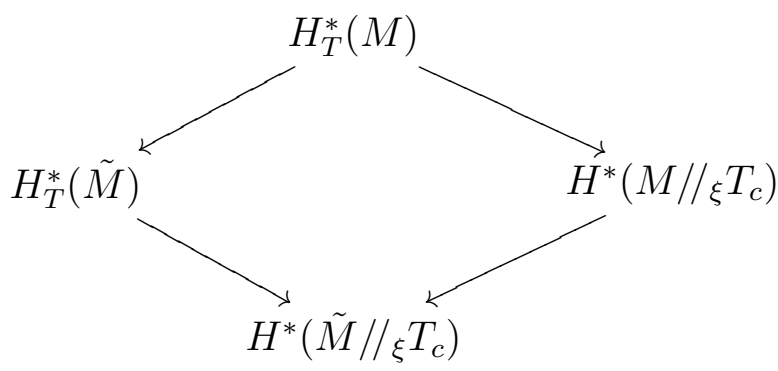

this implies that

$$
\kappa_{\tilde{M}, \xi}^{T}\left(\alpha \beta \mathcal{D}^{2}\right)\left[\tilde{M} / /{ }_{\xi} T_{c}\right]=\kappa_{M, \xi}^{T}\left(\alpha \beta \mathcal{D}^{2}\right)\left[M / /{ }_{\xi} T_{c}\right],
$$

and since $\xi$ is a regular value of $\mu_{T}$, the residue formula Theorem 3.1 gives us

Lemma 28 If the degrees of $\alpha, \beta \in H_{K}^{*}(M)$ add up to the real dimension of $M / / G$, then

$$
\kappa_{M, \xi}^{T}\left(\alpha \beta \mathcal{D}^{2}\right)\left[M / / \xi T_{c}\right]=\frac{n_{0}^{T}(-1)^{\ell}}{\operatorname{vol}(T)} \operatorname{res}\left(\mathcal{D}^{2} \sum_{F \in \mathcal{F}} \int_{F} \frac{i_{F}^{*}\left(\alpha \beta e^{\bar{\omega}-\xi}\right)(X)}{e_{F}}[d X]\right) .
$$


This means that to calculate the pairing $\kappa_{\tilde{M}}(\alpha \beta)[\tilde{M} / / G]$ it suffices to calculate the difference between

$$
\kappa_{\tilde{M}, \xi}^{T}\left(\alpha \beta \mathcal{D}^{2}\right)\left[\tilde{M} / / \xi T_{c}\right]
$$

and

$$
\kappa_{\tilde{M}}^{T}\left(\alpha \beta \mathcal{D}^{2}\right)\left[\tilde{M} / / T_{c}\right]
$$

(Note that (8.5) is just the special case of (8.4) when $\xi=0$.) This difference can be calculated using the version of nonabelian localization due to Guillemin and Kalkman [14] and independently to Martin [29, 30].

In fact since the construction of $\tilde{M}^{s s}$ from $M^{s s}$ described in Section 4 takes place in stages, it is actually easier to consider a single stage of the construction, when $\hat{M}$ is obtained by blowing $M$ up along the closure of $G Z_{R}^{s s}$ for a suitable reductive subgroup $R$ of $G$, after first resolving the singularities of $\overline{G Z_{R}^{s s}}$ (or equivalently, if we are only interested in the semistable points of $\hat{M}$, we can simply blow $M^{s s}$ up along $G Z_{R}^{s s}$ : see Remark (11). The argument which gave (8.3) also gives us

$$
\kappa_{\hat{M}, \xi}^{T}\left(\alpha \beta \mathcal{D}^{2}\right)\left[\hat{M} / / \xi T_{c}\right]=\kappa_{M}^{T}\left(\alpha \beta \mathcal{D}^{2}\right)\left[M / / \xi T_{c}\right]
$$

when $\xi$ is a regular value of $\mu_{T}$ and $k$ is chosen sufficiently large (depending on $\xi$ ). Let us choose $\xi \in \mathbf{t}^{*}$ to lie in a connected component $\Delta_{i}$ of the set of regular values of $\mu_{T}$ for which $0 \in \bar{\Delta}_{i}$. Let $\hat{\mu}$ and $\hat{\mu}_{T}$ be the moment maps for the actions of $K$ and $T$ on $\hat{M}$, and choose $\hat{\xi} \in \mathbf{t}^{*}$ to lie in the intersection of $\Delta_{i}$ and a connected component of the set of regular values of $\hat{\mu}_{T}$ which contains 0 in its closure. Because the choice of $k$, and hence also of the moment maps $\hat{\mu}$ and $\hat{\mu}_{T}$, depends on $\xi$, we cannot necessarily choose $\hat{\xi}=\xi$. However it suffices to calculate the difference

$$
\kappa_{\hat{M}, \xi}^{T}\left(\alpha \beta \mathcal{D}^{2}\right)\left[\hat{M} / / \xi T_{c}\right]-\kappa_{\hat{M}, \hat{\xi}}^{T}\left(\alpha \beta \mathcal{D}^{2}\right)\left[\hat{M} / / \hat{\xi} T_{c}\right],
$$

since combining this with (8.3) and (8.6) and iterating the calculation will give us the difference between $\kappa_{M, \xi}^{T}\left(\alpha \beta \mathcal{D}^{2}\right)\left[M / / \xi T_{c}\right]$ and $\kappa_{\tilde{M}, \tilde{\xi}}^{T}\left(\alpha \beta \mathcal{D}^{2}\right)\left[\tilde{M} / / \tilde{\xi}_{c} T_{c}\right]$ for any $\tilde{\xi}$ in a connected component of the set of regular values of $\tilde{\mu}_{T}$ which contains 0 in its closure. As 0 is itself a regular value of $\tilde{\mu}_{T}$, we can choose $\tilde{\xi}$ to be 0 and use Lemma 28 to calculate the pairing (8.1) which is our goal.

Remark 29 Notice also that since $\xi \in \Delta_{i}$ with $0 \in \bar{\Delta}_{i}$, we have

$$
M_{\xi, T}^{s s} \subseteq M_{0, T}^{s s}
$$

where $M_{0, T}^{s s}$ retracts $T$-equivariantly onto $\mu_{T}^{-1}(0)$, and similarly

$$
\hat{M}_{\hat{\xi}, T}^{s s} \subseteq \hat{M}_{0, T}^{s s}
$$

where $\hat{M}_{0, T}^{s s}$ retracts $T$-equivariantly onto $\hat{\mu}_{T}^{-1}(0)$. Thus by Remark 27 we do not need to resolve the singularities of the closure of $G Z_{R}^{s s}$ and construct the whole of $\hat{M}$ and $\tilde{M}$ in order to carry out these constructions; it suffices to consider the blow-up of $M^{s s}$ along $G Z_{R}^{s s}$ and iterations of this process. 
We know that the image $\hat{\mu}_{T}(\hat{M})$ of the moment map $\hat{\mu}_{T}$ is a convex polytope which is divided by walls of codimension one into subpolytopes whose interiors consist of regular values of $\hat{\mu}_{T}$. If $\eta \in H_{T}^{*}(\hat{M})$ there is no change in $\kappa_{\hat{M}, \xi}^{T}(\eta)\left[\hat{M} / / \xi T_{c}\right]$ as $\xi$ varies within a connected component of the set of regular values of $\hat{\mu}_{T}$, so it is enough to be able to calculate the change in $\kappa_{\hat{M}, \xi}^{T}(\eta)\left[\hat{M} / / \xi T_{c}\right]$ as $\xi$ crosses a wall of codimension one. Any such wall is of the form

$$
\hat{\mu}_{T}\left(\hat{M}_{1}\right)
$$

where $\hat{M}_{1}$ is a connected component of the fixed point set in $\hat{M}$ of a circle subgroup $T_{1}$ of $T$. The quotient group $T / T_{1}$ acts on $\hat{M}_{1}$, which is a symplectic submanifold of $\hat{M}$, and the restriction of the moment map $\hat{\mu}_{T}$ to $\hat{M}_{1}$ has an orthogonal decomposition

$$
\hat{\mu}_{T / T_{1}} \oplus \hat{\mu}_{T_{1}}
$$

where $\hat{\mu}_{T / T_{1}}: \hat{M}_{1} \rightarrow\left(\mathbf{t} / \mathbf{t}_{1}\right)^{*}$ is a moment map for the action of $T / T_{1}$ on $\hat{M}_{1}$, and $\mu_{T_{1}}$ is constant because the action of $T_{1}$ on $\hat{M}_{1}$ is trivial. Guillemin and Kalkman [14] show that the change in $\kappa_{\hat{M}, \xi}^{T}(\eta)\left[\hat{M} / /{ }_{\xi} T_{c}\right]$ as $\xi$ crosses a section of the wall $\hat{\mu}_{T}\left(\hat{M}_{1}\right)$ whose orthogonal projection onto $\left(\mathbf{t} / \mathbf{t}_{1}\right)^{*}$ contains a regular value $\xi_{1}$ for $\hat{\mu}_{T / T_{1}}$ is given by

$$
\kappa_{\hat{M}_{1}, \xi_{1}}^{T / T_{1}}\left(\operatorname{res}_{\hat{M}_{1}}(\eta)\right)\left[\hat{M}_{1} / / \xi_{1}\left(T / T_{1}\right)_{c}\right] .
$$

Here the residue operation

$$
\operatorname{res}_{\hat{M}_{1}}: H_{T}^{*}(\hat{M}) \rightarrow H_{T / T_{1}}^{*}\left(\hat{M}_{1}\right)
$$

is obtained by choosing a coordinate system $X=\left(X_{1}, \ldots, X_{\ell}\right)$ for $\mathbf{t}^{*}$ such that $X_{1}$ defines an integer basis for $\mathbf{t}_{1}^{*}$ and $\left(X_{2}, \ldots, X_{\ell}\right)$ is a coordinate system for the dual of a Lie algebra of a codimension one subtorus of $T$ whose intersection with $T_{1}$ is finite, and setting

$$
\operatorname{res}_{\hat{M}_{1}}(\eta)=\operatorname{res}_{X_{1}=0}\left(\frac{\left.\eta\right|_{\hat{M}_{1}}}{e_{\hat{M}_{1}}}\right)
$$

where $e_{\hat{M}_{1}}$ is the $T$-equivariant Euler class of the normal bundle to $\hat{M}_{1}$ in $\hat{M}$. We make sense of this residue as an element of $H_{T / T_{1}}^{*}\left(\hat{M}_{1}\right)$ by using $\left(X_{2}, \ldots, X_{\ell}\right)$ as coordinates on $\left(\mathbf{t} / \mathbf{t}_{1}\right)^{*}$ and writing

$$
e_{\hat{M}_{1}}=\prod_{i=1}^{\operatorname{codim} \hat{M}_{1}}\left(m_{i} X_{1}+e_{i}\right)
$$

where each $m_{i} \in \mathbb{Z} \backslash\{0\}$ is a weight for the action of $T_{1}$ on the normal bundle and each $e_{i}$ can be identified with an element of $H_{T / T_{1}}^{*}\left(\hat{M}_{1}\right)$.

Lemma 30 Let $\xi \in \mathbf{t}^{*}$ lie in a connected component $\Delta_{i}$ of the set of regular values of $\mu_{T}$ for which $0 \in \bar{\Delta}_{i}$, and let $\hat{\xi} \in \mathbf{t}^{*}$ lie in the intersection of $\Delta_{i}$ 
and a connected component of the set of regular values of $\hat{\mu}_{T}$ which contains 0 in its centre. Then in order to calculate the difference (8.7) the only wall crossing terms we need to consider correspond to components $\hat{M}_{1}$ of fixed point sets of circle subgroups $T_{1}$ satisfying

$$
\emptyset \neq \pi\left(\hat{M}_{1}\right) \cap M^{s s} \subseteq G Z_{R}^{s s}
$$

so that $\hat{M}_{1}$ is contained in the exceptional divisor of $\pi: \hat{M} \rightarrow M$.

Proof: From Remark [10] we see that by choosing $k$ sufficiently large we can assume that (after scaling by $1 / k)$ the wall $\hat{\mu}_{T}\left(\hat{M}_{1}\right)$ is contained in an arbitrarily small neighbourhood of $\mu_{T}\left(\pi\left(\hat{M}_{1}\right)\right)$. Notice that $\pi\left(\hat{M}_{1}\right)$ is contained in a connected component of the fixed point set for the action of $T_{1}$ on $M$, and hence $\mu_{T}\left(\pi\left(\hat{M}_{1}\right)\right)$ is contained in a wall for the action of $T$ on $M$ with moment map $\mu_{T}$. Recall that we have chosen $\xi \in \mathbf{t}^{*}$ (respectively $\hat{\xi} \in \mathbf{t}^{*}$ ) to lie in a connected component of the set of regular values of $\mu_{T}$ (respectively $\hat{\mu}_{T}$ ) containing 0 in its closure. It follows that for $k \gg 0$ it is possible to reach $\xi$ from $\hat{\xi}$ by crossing only those walls $\hat{\mu}_{T}\left(\hat{M}_{1}\right)$ for the action of $T$ on $\hat{M}$ for which $\mu_{T}\left(\pi\left(\hat{M}_{1}\right)\right)$ contains 0 , and in particular the constant value taken by $\mu_{T_{1}} \circ \pi$ is 0 . Moreover we can always assume that the $\xi_{1}$ which appears in (8.8) lies in a connected component of the set of regular values of $\hat{\mu}_{T / T_{1}}$ whose closure contains 0 .

Suppose $\pi\left(\hat{M}_{1}\right) \cap M^{s s}=\emptyset$. Since $T_{1}$ fixes $\hat{M}_{1}$, its image $\pi\left(\hat{M}_{1}\right)$ is contained in a $T_{1}$-fixed point component in $M$. If the wall given by this component does not pass through 0 , we do not need to cross the wall determined by $\hat{M}_{1}$ because it is a wall far away from 0 in our scale.

Let $B \subseteq \mathbf{t}=\operatorname{Lie}(T)$ be a small ball containing 0 . On $\mu_{T}^{-1}(B)$, we may choose an equivariant differential form representing $\left.\mathcal{D}\right|_{\mu_{T}^{-1}(B)}$, supported in $\mu_{T}^{-1}(B) \cap M^{s s}$. If $\pi\left(\hat{M}_{1}\right) \cap M^{s s}=\emptyset$ and the wall for $\pi\left(\hat{M}_{1}\right)$ passes through 0 , we may assume $\xi_{1} \in B$ and the wall crossing term

$$
\kappa_{\hat{M}_{1}, \xi_{1}}^{T / T_{1}}\left(\operatorname{res}_{\hat{M}_{1}}\left(\alpha \beta \mathcal{D}^{2}\right)\right)\left[\hat{M}_{1} / / \xi_{1}\left(T / T_{1}\right)_{c}\right]
$$

vanishes since $\left.\alpha \beta \mathcal{D}^{2}\right|_{\pi\left(\hat{M}_{1}\right) \cap \mu_{T}^{-1}\left(\xi_{1}\right)}=0$.

If $\pi\left(\hat{M}_{1}\right) \cap M^{s s}$ is non-empty but not contained in $G Z_{R}^{s s}$, then the wall $\hat{\mu}_{T}\left(\hat{M}_{1}\right)$ is determined by the image of $\hat{M}_{1} \backslash \pi^{-1}\left(\overline{G Z_{R}^{s s}}\right)$ under the moment map $\hat{\mu}_{T}$ which is determined by the linearization of the action on $\hat{M}$ given by the induced action on the line bundle $\pi^{*} L^{\otimes k} \otimes \mathcal{O}(-E)$. As above we can assume that $0 \in \mu_{T}\left(\pi\left(\hat{M}_{1}\right)\right)$ and hence the constant value taken on $\pi\left(\hat{M}_{1}\right)$ by the $T_{1}$-moment map $\mu_{T_{1}}$ is 0 . This means that the induced action of $T_{1}$ on the restriction of $L$ to $\pi\left(\hat{M}_{1}\right)$ is trivial so the same is true for the induced action of $T_{1}$ on the restriction of $\pi^{*} L^{\otimes k}$ to $\hat{M}_{1}$. Moreover since $\hat{M}_{1} \backslash \pi^{-1}\left(\overline{G Z_{R}^{s s}}\right)$ does not meet the exceptional divisor $E$ we have

$$
\left.\left.\mathcal{O}(-E)\right|_{\hat{M}_{1} \backslash \pi^{-1}\left(\overline{G Z_{R}^{s s}}\right)} \cong \mathcal{O}\right|_{\hat{M}_{1} \backslash \pi^{-1}\left(\overline{G Z_{R}^{s s}}\right)}
$$


and the induced action of $T_{1}$ on this is trivial since $T_{1}$ acts trivially on $\hat{M}_{1}$. Hence the induced action of $T_{1}$ on the restriction of $\pi^{*} L^{\otimes k} \otimes \mathcal{O}(-E)$ to $\hat{M}_{1} \backslash \pi^{-1}\left(\overline{G Z_{R}^{s s}}\right)$ is trivial, and hence the constant value taken by $\hat{\mu}_{T_{1}}$ on $\hat{M}_{1}$ is 0 . Therefore the wall $\hat{\mu}_{T}\left(\hat{M}_{1}\right)$ passes through 0 , and hence need not be crossed.

Hence the only wall crossing terms we need to consider are for the fixed point sets $\hat{M}_{1}$ such that $\emptyset \neq \pi\left(\hat{M}_{1}\right) \cap M^{s s} \subseteq G Z_{R}^{s s}$ as required.

Example 31 Let us consider again the action of $G=S L(2)$ on $M=\mathbb{P}_{n}$ when $n$ is even. We need to blow $M$ up along the closure of the $G$-orbit of the unique $T$-fixed point $p_{0}$ in $\mu^{-1}(0)$ in order to obtain $\tilde{M}$. The only $T$-fixed point in $G p_{0}$ is $p_{0}$ itself and the weights of the induced action on the fiber $\mathbb{P}_{n-3}$ over $p_{0}$ in the exceptional divisor of $\tilde{M}$ are $-n, 2-n, \ldots, n-2, n$ with $0,-2,2$ omitted. If as in Example 26] $\eta=q\left(\xi, \zeta^{2}\right) \in H_{K}^{*}(M)$ has degree $2(n-3)$ then

$$
\kappa_{\tilde{M}}(\eta)[\tilde{M} / / G]=-\frac{1}{2} \kappa_{\tilde{M}}\left(\eta \mathcal{D}^{2}\right)\left[\tilde{M} / / T_{c}\right]
$$

and we can write

$\kappa_{\tilde{M}}\left(\eta \mathcal{D}^{2}\right)\left[\tilde{M} / / T_{c}\right]=\left(\kappa_{\tilde{M}}\left(\eta \mathcal{D}^{2}\right)\left[\tilde{M} / / T_{c}\right]-\kappa_{\tilde{M}}\left(\eta \mathcal{D}^{2}\right)\left[\tilde{M} / /{ }_{\xi} T_{c}\right]\right)+\kappa_{\tilde{M}}\left(\eta \mathcal{D}^{2}\right)\left[\tilde{M} / / \xi T_{c}\right]$

where $\xi$ is a positive number between 0 and 1 .

Notice that $\kappa_{\tilde{M}}\left(\eta \mathcal{D}^{2}\right)\left[\tilde{M} / /{ }_{\xi} T_{c}\right]=\kappa_{M}\left(\eta \mathcal{D}^{2}\right)\left[M / / \xi T_{c}\right]$, which is given by summing up the wall crossing terms for the components of the fixed point set with positive moment map values. Hence,

$$
\kappa_{\tilde{M}}\left(\eta \mathcal{D}^{2}\right)\left[\tilde{M} / /{ }_{\xi} T_{c}\right]=-2 \operatorname{res}_{X=0}\left(\sum_{j: 0<n-2 j \leq n} \frac{q\left((n-2 j) X, X^{2}\right)}{(2 X)^{n-2} \prod_{k \neq j}(k-j)}\right) .
$$

On the other hand, the difference $\kappa_{\tilde{M}}\left(\eta \mathcal{D}^{2}\right)\left[\tilde{M} / /{ }_{0} T_{c}\right]-\kappa_{\tilde{M}}\left(\eta \mathcal{D}^{2}\right)\left[\tilde{M} / /{ }_{\xi} T_{c}\right]$ is given by the wall crossing terms for the components of the fixed point set over $p_{0}$ with positive moment map values. Hence the difference is

$$
2 \operatorname{res}_{X=0}\left(\sum_{j: 2<n-2 j \leq n} \frac{q\left(0, X^{2}\right)}{2^{n-3} X^{n-2}(n-2 j) \prod_{k \neq j, 0}(k-j)}\right)
$$

Note however that because $n$ is even the residue is always 0 . Therefore, we get

$$
\kappa_{\tilde{M}}(\eta)[\tilde{M} / / G]=\operatorname{res}_{X=0}\left(\sum_{j: 0<n-2 j \leq n} \frac{q\left((n-2 j) X, X^{2}\right)}{(2 X)^{n-2} \prod_{k \neq j}(k-j)}\right) .
$$

Let us consider in more detail the walls $\hat{\mu}_{T}\left(\hat{M}_{1}\right)$ and the wall-crossing terms (8.9) in the general case. Consider the subset

$$
L=\left\{g \in G: T_{1} \subseteq g R g^{-1}\right\}
$$


of $G$. By (8.10) of [26], $L$ is the disjoint union of finitely many double cosets for the left $N_{0}^{T_{1}}$ action and the right $N^{R}$ action

$$
L=\bigsqcup_{1 \leq i \leq m} N_{0}^{T_{1}} g_{i} N^{R}
$$

where $N_{0}^{T_{1}}$ is the identity component of the normalizer of $T_{1}$ in $G$ and $N^{R}$ is the normalizer of $R$ in $G$. Since $G Z_{R}^{s s} \cong G \times_{N^{R}} Z_{R}^{s s}$ it follows that the $T_{1}$-fixed point set in $G Z_{R}^{s s}$ is the disjoint union

$$
\bigsqcup_{1 \leq i \leq m} N_{0}^{T_{1}} Z_{R_{i}}^{s s}
$$

where $R_{i}=g_{i} R g_{i}^{-1} \supset T_{1}$.

Definition 32 Let $W_{i, j} \rightarrow N_{0}^{T_{1}} Z_{R_{i}}^{s s}$ for $1 \leq j \leq l_{i}$ be the $T_{1}$-eigenbundle of the restriction to $N_{0}^{T_{1}} Z_{R_{i}}^{s s}$ of the normal bundle to $G Z_{R}^{s s}$ on which $T_{1}$ acts with weight $\beta_{i, j}$.

Then $\bigsqcup_{i, j} \mathbb{P} W_{i, j}$ is the $T_{1}$ fixed point set in $\pi^{-1}\left(G Z_{R}^{s s}\right)$, and we have proved

Lemma 33 Let $\hat{M}_{1}$ be a component of a fixed point set of a circle subgroup $T_{1}$ satisfying $\emptyset \neq \pi\left(\hat{M}_{1}\right) \cap M^{s s} \subseteq G Z_{R}^{s s}$ as in Lemma [30. Then

$$
\hat{M}_{1} \cap \pi^{-1}\left(M^{s s}\right)=\mathbb{P} W_{i, j}
$$

for some $i, j$, and the corresponding wall crossing term (8.9) is

$$
\int_{\mathbb{P} W_{i, j} / / \xi_{1}\left(T / T_{1}\right)_{c}} \kappa_{\mathbb{P} W_{i, j}, \xi_{1}}^{T / T_{1}}\left(\operatorname{res}_{X_{1}=0} \frac{\alpha \beta \mathcal{D}^{2}}{e_{\mathbb{P} W_{i, j}}}\right)
$$

where $e_{\mathbb{P} W_{i, j}}$ is the T-equivariant Euler class of the normal bundle to $\mathbb{P} W_{i, j}$ in $\hat{M}$.

Remark $34 \hat{M}_{1}$ is fixed by $T_{1}$, so its images under $\hat{\mu}$ and $\mu \circ \pi$ are fixed by $T_{1}$, and hence they are contained in the Lie algebra of the centralizer of $T_{1}$. As $T_{1}$ is a circle it has no nontrivial orientation preserving automorphisms, so the connected component of this centralizer is just $N_{0}^{T_{1}} \cap K$. In fact when $K$ is connected any such centralizer is connected, so the centralizer of $T_{1}$ in $K$ is $N_{0}^{T_{1}} \cap K$ and the centralizer of $T_{1}$ in $G$ is $N_{0}^{T_{1}}$. Also the $T_{1}$ component of $\hat{\mu}$ is constant on $\hat{M}_{1}$; let us call it $\xi_{2}$. Then it follows that

$$
\hat{\mu}_{N_{0}^{T_{1}} / T_{1}}^{-1}\left(\xi_{1}\right) \cap \hat{M}_{1}=\hat{\mu}^{-1}\left(\xi_{1}+\xi_{2}\right) \cap \hat{M}_{1} .
$$

Note that $\xi_{1}$ and $\xi_{2}$ can be taken to be arbitrarily close to 0 , so that $\hat{\mu}^{-1}\left(\xi_{1}+\right.$ $\xi_{2}$ ) lies over semistable points in $M$ (cf. Remark 8.3). 
We are looking at the evaluation of $\operatorname{res}_{\hat{M}_{1}} \alpha \beta \mathcal{D}^{2}$ on

$$
\left(\hat{\mu}_{T / T_{1}}^{-1}\left(\xi_{1}\right) \cap \hat{M}_{1}\right) /\left(T / T_{1}\right) .
$$

This can be calculated inductively as follows. Let

$$
\widetilde{\mathbb{P} W}_{i, j} / / N_{0}^{T_{1}}=\widetilde{\mathbb{P} W}_{i, j} / /\left(N_{0}^{T_{1}} /\left(T_{1}\right)_{c}\right)
$$

be the partial desingularization of $\mathbb{P} W_{i, j} / / N_{0}^{T_{1}}$. By (8.3), we have

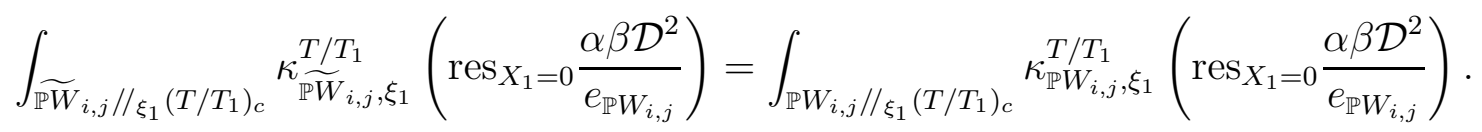

Since $\operatorname{dim} \hat{M}_{1}<\operatorname{dim} M$ and $\operatorname{dim}\left(N_{0}^{T_{1}} /\left(T_{1}\right)_{c}\right)<\operatorname{dim} G$, we can calculate the difference between the wall crossing term above and

$$
\int_{\mathbb{P} W_{i, j} / /\left(T / T_{1}\right)_{c}} \kappa_{\mathbb{P} W_{i, j}}^{T / T_{1}}\left(\operatorname{res}_{X_{1}=0} \frac{\alpha \beta \mathcal{D}^{2}}{e_{\mathbb{P} W_{i, j}}}\right)
$$

inductively using the same procedure.

Now, as at (8.1), we find that (8.10) is equal up to a scalar factor analogous to $\frac{n_{0}(-1)^{n_{+}}}{n_{0}^{T}|W|}$ to

$$
\int_{\widetilde{P W}_{i, j} / / N_{0}^{T_{1}}} \kappa_{\mathbb{P}_{i, j}}\left(\operatorname{res}_{X_{1}=0} \frac{\alpha \beta \mathcal{D}^{2}}{e_{\mathbb{P} W_{i, j}}\left(\mathcal{D}_{N_{0}^{T_{1}}}\right)^{2}}\right)
$$

where $\mathcal{D}_{N_{0}^{T_{1}}}$ is the product of the positive roots of $N_{0}^{T_{1}}$.

Remark 35 The wall crossing term (8.9) is an integral over the quotient $\hat{M}_{1} / / \xi_{1}\left(T / T_{1}\right)_{c}=\hat{\mu}_{T}^{-1}\left(\xi_{1}+\xi_{2}\right) \cap \hat{M}_{1} / T$. Since the intersection of $\hat{M}_{1}$ with $\pi^{-1}\left(M^{s s}\right)$ is a projective bundle over $N_{0}^{T_{1}} Z_{R_{1}}^{s s}$, it is easier to describe quotients of $\hat{M}_{1}$ by $N_{0}^{T_{1}}$ than by $T$, and we can use (1.6) to relate integrals over quotients of $\hat{M}_{1}$ by $T$ to integrals over quotients of $\hat{M}_{1}$ by $N_{0}^{T_{1}}$. Unfortunately, however, the quotient

$$
\hat{M}_{1} / / \xi_{1}\left(N_{0}^{T_{1}} /\left(T_{1}\right)_{c}\right)=\hat{\mu}^{-1}\left(\xi_{1}+\xi_{2}\right) \cap \hat{M}_{1} /\left(N_{0}^{T_{1}} \cap K\right)
$$

is only well defined if $\xi_{1}$ is centralized by $N_{0}^{T_{1}}$, and this is not necessarily the case. To overcome this problem, we can use induction to relate our integral over $\hat{M}_{1} / / \xi_{1}\left(T / T_{1}\right)_{c}$ to an integral over the partial desingularization of $\hat{M}_{1} / / N_{0}^{T_{1}}$, as above, or indeed to an integral over the partial desingularization of $M_{1} / /{ }_{\zeta} N_{0}^{T_{1}}$ for any $\zeta$ in the Lie algebra of the centre of $N_{0}^{T_{1}} \cap K$. In what follows we shall assume for simplicity that $\zeta=0$, but the same argument will work for any central $\zeta$. In particular if $\xi_{1}$ itself is central then we can simply work with $\hat{M}_{1} / / \xi_{1} N_{0}^{T_{1}}$ and no partial desingularization is required. 
Remark 36 At this point it is useful to recall from [26, Lemma 7.8 that the fibre at $x \in Z_{R}^{s s}$ of the restriction $\pi: \hat{M}^{s s} \rightarrow M^{s s}$ of $\pi$ to $\hat{M}^{s s}$ is the semistable stratum of the projective space associated to the normal to $G Z_{R}^{s s}$ in $M^{s s}$ at $x$ with respect to the representation of $R$ on the normal space. Since $R \subseteq N^{R} \subset G$ and semistability for some reductive group implies semistability for any reductive subgroup, it follows that in such a fibre semistability for $R$, for $N^{R}$ and for $G$ are all equivalent. Similarly (cf. Remark 34) in the intersection $\mathbb{P}\left(W_{i, j}\right)_{x}$ of $\hat{M}_{1}=\mathbb{P} W_{i, j}$ with the fibre of $\pi$ at $x \in Z_{R_{i}}^{s s}$, semistability for $R_{i} \cap N_{0}^{T_{1}}$, for $N^{R_{i}} \cap N_{0}^{T_{1}}$ and for $N_{0}^{T_{1}}$ are all equivalent.

Recall that $G Z_{R}^{s s} \cong G \times{ }_{N^{R}} Z_{R}^{s s}$ and hence

$$
N_{0}^{T_{1}} Z_{R_{i}}^{s s} \cong N_{0}^{T_{1}} \times_{N_{0}^{T_{1}} \cap N^{R_{i}}} Z_{R_{i}}^{s s}
$$

and so

$$
\begin{gathered}
\mathbb{P} W_{i, j} \cong N_{0}^{T_{1}} \times\left._{N_{0}^{T_{1}} \cap N^{R_{i}}} \mathbb{P} W_{i, j}\right|_{Z_{R_{i}}^{s s}} \\
\widetilde{\mathbb{P} W}_{i, j} \cong N_{0}^{T_{1}} \times\left._{N_{0}^{T_{1}} \cap N^{R_{i}}} \widetilde{\mathbb{P} W_{i, j}}\right|_{Z_{R_{i}}^{s s}} .
\end{gathered}
$$

It then follows immediately from Remark 36 that

$$
\widetilde{\mathbb{P W}}_{i, j} / /\left.N_{0}^{T_{1}} \cong \widetilde{\mathbb{P} W}_{i, j}\right|_{Z_{R_{i}}^{s s}} / / N_{0}^{T_{1}} \cap N^{R_{i}}
$$

Lemma $37 R_{i}\left(N_{0}^{T_{1}} \cap N^{R_{i}}\right)$ is a subgroup of finite index in $N^{R_{i}}$.

Proof: We modify the proof of [26], Lemma 8.10. First note that each subgroup $R_{i}$ and $N_{0}^{T_{1}}$ and $N^{R_{i}}$ of $G$ is the complexification of its intersection with the maximal compact subgroup $K$ of $G$, and so it suffices to show that $\left(R_{i} \cap K\right)\left(N_{0}^{T_{1}} \cap N^{R_{i}} \cap K\right)$ has finite index in $N^{R_{i}} \cap K$.

Consider the action of $R_{i} \cap K$ on the homogeneous space $\left(N^{R_{i}} \cap K\right) / T_{1}$. If $k \in N^{R_{i}} \cap K$ then the stabilizer in $R_{i} \cap K$ of the coset $k T_{1}$ is

$$
\left\{s \in R_{i} \cap K \mid s k T_{1}=k T_{1}\right\}=R_{i} \cap k T_{1} k^{-1}
$$

since $T_{1} \subseteq R_{i}$ and $k$ normalizes $T_{1}$. But only finitely many conjugacy classes of subgroups of $R_{i} \cap K$ can occur as stabilizers of elements of $\left(N^{R_{i}} \cap K\right) / T_{1}$ (see [33]). Therefore there exist $k_{1}, \ldots, k_{m} \in N^{R_{i}} \cap K$ such that if $k \in N^{R_{i}} \cap K$ then

$$
k T_{1} k^{-1}=r k_{j} T_{1} k_{j}^{-1} r^{-1}
$$

for some $r \in R_{i} \cap K$ and $j \in\{1, \ldots, n\}$, and hence

$$
\begin{gathered}
k \in\left(R_{i} \cap K\right) k_{j}\left(N^{T_{1}} \cap N^{R_{i}} \cap K\right) \\
=k_{j}\left(R_{i} \cap K\right)\left(N^{T_{1}} \cap N^{R_{i}} \cap K\right)
\end{gathered}
$$

since $k_{j}$ normalizes $R_{i} \cap K$. Since $N_{0}^{T_{1}}$ has finite index in $N^{T_{1}}$, the result follows. 
Remark 38 It follows immediately that the connected component $N_{0}^{R_{i}}$ of $N^{R_{i}}$ is equal to the connected component $R_{i}\left(N_{0}^{T_{1}} \cap N^{R_{i}}\right)_{0}$ of $R_{i}\left(N_{0}^{T_{1}} \cap N^{R_{i}}\right)$.

Corollary 39 There is a natural isomorphism

$$
Z_{R_{i}} / /\left(N_{0}^{T_{1}} \cap N^{R_{i}}\right)_{0} \cong Z_{R_{i}} / / N_{0}^{R_{i}}
$$

and finite-to-one surjections

$$
Z_{R_{i}} / / N_{0}^{R_{i}} \rightarrow Z_{R_{i}} / / N^{R_{i}}
$$

and

$$
Z_{R_{i}} / / N_{0}^{R_{i}} \rightarrow Z_{R_{i}} / / N_{0}^{T_{1}} \cap N^{R_{i}}
$$

Proof: This follows immediately because the linear action of $R_{i}$ on $Z_{R_{i}}$ is trivial.

We have reduced the calculation of the wall crossing term (8.9) to the calculation of the integral (8.11) over $\widetilde{\mathbb{P} W}_{i, j} / / N_{0}^{T_{1}}$ and from (8.12) we know that

$$
\widetilde{\mathbb{P} W}_{i, j} / /\left.N_{0}^{T_{1}} \cong \widetilde{\mathbb{P} W}_{i, j}\right|_{Z_{R_{i}}^{s s}} / / N_{0}^{T_{1}} \cap N^{R_{i}}
$$

We have a surjection

$$
\left.\widetilde{\mathbb{P} W}_{i, j}\right|_{Z_{R_{i}}^{s s}} / / N_{0}^{T_{1}} \cap N^{R_{i}} \stackrel{\Psi}{\longrightarrow} Z_{R_{i}} / / N_{0}^{T_{1}} \cap N^{R_{i}}
$$

Lemma 40 The fibre of $\Psi$ is the partial desingularization

$$
\left.\widetilde{\mathbb{P} W}_{i, j}\right|_{x} / / \operatorname{Stab}(x) \cap N_{0}^{T_{1}} \cap N^{R_{i}}
$$

of the quotient of the projective space $\left.\mathbb{P} W_{i, j}\right|_{x}$ by $\operatorname{Stab}(x) \cap N_{0}^{T_{1}} \cap N^{R_{i}}$.

Proof: This follows since the centre of the blowup required at any stage of the construction of $\widetilde{\mathbb{P} W}_{i, j} / / N_{0}^{T_{1}}$ intersects the fibre at $x \in Z_{R_{i}}^{s s}$ in the centre of the corresponding blowup required for the construction of the partial desingularization

$$
\left.\widetilde{\mathbb{P} W}_{i, j}\right|_{x} / / \operatorname{Stab}(x) \cap N_{0}^{T_{1}} \cap N^{R_{i}}
$$

(cf. the proofs of [27] Lemma 1.16 and Proposition 1.20).

We can now perform the integration needed over

$$
\left.\widetilde{\mathbb{P} W}_{i, j}\right|_{Z_{R_{i}}^{s s}} / / N_{0}^{T_{1}} \cap N^{R_{i}}
$$

by first integrating over the fibres of $\Psi$. This involves integrals over partial desingularizations

$$
\left.\widetilde{\mathbb{P} W}_{i, j}\right|_{x} / / \operatorname{Stab}(x) \cap N_{0}^{T_{1}} \cap N^{R_{i}}
$$

and

$$
Z_{R_{i}} / / N_{0}^{T_{1}} \cap N^{R_{i}}
$$

which can be calculated inductively. 
Remark 41 (i) Recall from Corollary 39] that there is a natural surjection $Z_{R_{i}} / / N_{0}^{R_{i}} \stackrel{\Phi}{\longrightarrow} Z_{R_{i}} / / N^{R_{i}} \cap N_{0}^{T_{1}}$ such that if $\eta \in H^{*}\left(Z_{R_{i}} / / N_{0}^{T_{1}} \cap N^{R_{i}}\right)$ then

$$
\int_{Z_{R_{i}} / / N^{R_{i}} \cap N_{0}^{T_{1}}} \eta=\frac{1}{c} \int_{Z_{R_{i}} / / N_{0}^{R_{i}}} \Phi^{*} \eta
$$

where $c$ is the order of

$$
\frac{\operatorname{Stab}_{R_{i}\left(N_{0}^{T_{1}} \cap N^{R_{i}}\right)}(x)}{\operatorname{Stab}_{N_{0}^{R_{i}}}(x)}
$$

for a generic $x \in Z_{R_{i}}^{s s}$.

(ii) The connected component of $\operatorname{Stab}(x)$ for $x \in Z_{R_{i}}^{s s}$ is $R_{i}$ so $N_{0}^{T_{1}} \cap R_{i}$ is a subgroup of finite index in $\operatorname{Stab}(x) \cap N_{0}^{T_{1}} \cap N^{R_{i}}$, and hence as in (i) we can reduce integrals over

$$
\left.\widetilde{\mathbb{P} W}_{i, j}\right|_{x} / / \operatorname{Stab}(x) \cap N_{0}^{T_{1}} \cap N^{R_{i}}
$$

to integrals over

$$
\left.\widetilde{\mathbb{P} W}_{i, j}\right|_{x} / / R_{i} \cap N_{0}^{T_{1}}
$$

(iii) We have assumed for simplicity that the blowup along $G Z_{R_{i}}^{s s}$ is the first stage of the construction of $\tilde{M} / / G$ from $M / / G$. If instead we are at a later stage of the procedure, we need to replace $Z_{R_{i}} / / N^{R_{i}}$ by its partial desingularization $\tilde{Z}_{R_{i}} / / N^{R_{i}}$ throughout; cf. [26], Lemma 8.8.

Recall that in order to calculate the pairing $\kappa_{\tilde{M}}(\alpha \beta)[\tilde{M} / / G]$, by $(8.1)$ and Lemma 8.2 it suffices to calculate the difference

$$
\kappa_{\tilde{M}, \xi}^{T}\left(\alpha \beta \mathcal{D}^{2}\right)\left[\tilde{M} / / \xi T_{c}\right]-\kappa_{\tilde{M}}^{T}\left(\alpha \beta \mathcal{D}^{2}\right)\left[\tilde{M} / / T_{c}\right]
$$

which is a sum of differences of the form

$$
\kappa_{\hat{M}, \xi}^{T}\left(\alpha \beta \mathcal{D}^{2}\right)\left[\hat{M} / /{ }_{\xi} T_{c}\right]-\kappa_{\hat{M}, \hat{\xi}}^{T}\left(\alpha \beta \mathcal{D}^{2}\right)\left[\hat{M} / /{ }_{\hat{\xi}} T_{c}\right]
$$

fo suitable $\xi$ and $\hat{\xi}$. Recall also that these differences are in turn sums of wall crossing terms (8.9)

$$
\kappa_{\hat{M}_{1}, \xi_{1}}^{T / T_{1}}\left(\operatorname{res}_{\hat{M}_{1}}\left(\alpha \beta \mathcal{D}^{2}\right)\right)\left[\hat{M}_{1} / / \xi_{1}\left(T / T_{1}\right)_{c}\right]=\int_{\mathbb{P} W_{i, j} / \xi_{1}\left(T / T_{1}\right)_{c}} \kappa_{\mathbb{P} W_{i, j}, \xi_{1}}^{T / T_{1}}\left(\operatorname{res}_{X_{1}=0} \frac{\alpha \beta \mathcal{D}^{2}}{e_{\mathbb{P} W_{i, j}}}\right),
$$

and the difference between any such wall crossing term and the corresponding integral (8.11)

$$
\int_{\widetilde{\mathbb{P} W}_{i, j} / / N_{0}^{T_{1}}} \kappa_{\widetilde{P}_{i, j}}\left(\operatorname{res}_{X_{1}=0} \frac{\alpha \beta \mathcal{D}^{2}}{e_{\mathbb{P} W_{i, j}}\left(\mathcal{D}_{N_{0}^{T_{1}}}\right)^{2}}\right)
$$


over the partial desingularization of $\mathbb{P} W_{i, j} / / N_{0}^{T_{1}}$ can be calculated inductively. Finally we have just observed that we can perform this last integral by integrating over the fibres of the map

$$
\Psi: \widetilde{\mathbb{P} W}_{i, j} / / N_{0}^{T_{1}} \rightarrow Z_{R_{i}} / / N_{0}^{T_{1}} \cap N^{R_{i}}
$$

and the integral over the fibre of $\Psi$ at a point represented by $x \in Z_{R_{i}}^{s s}$ reduces to an integral over the partial desingularization $\left.\widetilde{\mathbb{P} W}_{i, j}\right|_{x} / / R_{i}$ of the GIT quotient of the projective space $\left.\mathbb{P} W_{i, j}\right|_{x}$ by $R_{i}$, while the resulting integral over the base space $Z_{R_{i}} / / N_{0}^{T_{1}} \cap N^{R_{i}}$ can be pulled back to an integral over $Z_{R_{i}} / / N^{R_{i}}$. We can use induction and the residue formula Theorem 3.1(i) to calculate integrals over $Z_{R_{i}} / / N^{R_{i}}$ of any cohomology classes represented by equivariant cohomology classes on $Z_{R_{i}}$, so we can calculate (8.11) provided that we are able to represent the cohomology class on $Z_{R_{i}} / / N^{R_{i}}$ obtained by integrating

$$
\kappa_{\mathbb{P} W_{i, j}}\left(\operatorname{res}_{X_{1}=0} \frac{\alpha \beta \mathcal{D}^{2}}{e_{\mathbb{P} W_{i, j}}\left(\mathcal{D}_{N_{0}^{T_{1}}}\right)^{2}}\right)
$$

over the fibres of $\Psi$ by an equivariant cohomology class on $Z_{R_{i}}$. But if $x \in Z_{R_{i}}^{s s}$ then we can use induction and the residue formula Theorem 3.1(i) to express the integral over $\left.\widetilde{\mathbb{P} W}_{i, j}\right|_{x} / / R_{i}$ of (8.15) in terms of residues of integrals over fibres at $x$ of projective subbundles of $\left.\mathbb{P} W_{i, j}\right|_{R_{i}} ^{s s}$ which extend naturally to projective bundles over $Z_{R_{i}}$. Such integrals can be represented by equivariant cohomology classes on $Z_{R_{i}}$ using the following lemma, which therefore completes our procedure for calculating $\kappa_{\tilde{M}}(\alpha \beta)[\tilde{M} / / G]$.

Lemma 42 Let $E$ be a rank $r$ complex vector bundle over a manifold $M$. Then

$$
\int_{\mathbf{P} E} \eta=\operatorname{Res}_{y=0} \int_{M} \frac{\eta}{p(y)}
$$

where $p(y)=y^{r}+c_{1}(E) y^{r-1}+\ldots+c_{r}(E)$.

Proof: This follows from standard arguments in algebraic topology. Here we give a simple argument. Let $P$ denote $\mathbf{P}(E)$, and let $\pi: P \rightarrow M$ be the projection. Then $H^{*}(P)$ is isomorphic to the quotient of the polynomial ring $H^{*}(M)[y]$ by the ideal generated by $p(y)$ as above. For any cohomology class $\eta \in H^{*}(P)$, we have $\eta=\sum_{i=0}^{r-1} \pi^{*} \beta_{i} y^{i}$ for suitable classes $\beta_{i} \in H^{*}(M)$, where we have identified $y$ with the first Chern class of the hyperplane line bundle $L \rightarrow P$. Then

$$
\frac{1}{p(y)}=\frac{1}{y^{r}\left(1+\frac{c_{1}(E)}{y}+\ldots+\frac{c_{r}(E)}{y^{r}}\right)}=\sum_{k \geq 0} \frac{(-1)^{k}}{y^{r}}\left(\frac{c_{1}(E)}{y}+\ldots+\frac{c_{r}(E)}{y^{r}}\right)^{k}
$$

and so

$$
\int_{P} \eta=\int_{M} \pi^{*} \beta_{n-1}=\operatorname{Res}_{y=0} \int_{M} \frac{\eta}{p(y)}
$$




\section{Witten's integral}

In the case when 0 is a regular value of the moment map $\mu$ Witten relates the intersection pairings of two classes $\kappa_{M}(\alpha), \kappa_{M}(\beta)$ of complementary degrees in $H^{*}(M / / G)$ coming from $\alpha, \beta \in H_{K}^{*}(M)$ to the asymptotic behaviour of the integral $\mathcal{I}^{\epsilon}\left(\alpha \beta e^{i \bar{\omega}}\right)$ given by

$$
\mathcal{I}^{\epsilon}\left(\eta e^{i \bar{\omega}}\right)=\frac{1}{(2 \pi)^{s} \operatorname{vol}(K)} \int_{X \in \mathbf{k}}[d X] e^{-\epsilon<X, X>/ 2} \int_{M} \eta(X) e^{i \omega} e^{i \mu(X)},
$$

where as before $\bar{\omega}=\omega+\mu$. He expresses the integral as a sum of local contributions, one of which reduces to the intersection pairing required while the rest tend to 0 exponentially fast as $\epsilon$ tends to 0 .

Even when 0 is not a regular value of $\mu$, Witten's integral $\mathcal{I}^{\epsilon}\left(\eta e^{i \bar{\omega}}\right)$ decomposes into the sum of a term $\mathcal{I}_{0}^{\epsilon}\left(\eta e^{i \bar{\omega}}\right)$ determined by the action of $K$ on an arbitrarily small neighbourhood of $\mu^{-1}(0)$, and other terms which tend to zero exponentially fast as $\epsilon \rightarrow 0$. We shall see that there is a residue formula for $\mathcal{I}_{0}^{\epsilon}\left(\eta e^{i \bar{\omega}}\right)$ which again is a sum over components of the fixed point set of $T$ on $M$. This residue formula is related to the formulas for pairings in the intersection homology of $M / / G$ given in previous sections, but it is not in general a polynomial in $\epsilon$; instead it is a polynomial in $\sqrt{\epsilon}$, as has been proved by Paradan (see [36] Cor.5.2).

In Sections 4 and 7 of [20] it is proved that the integral $\mathcal{I}^{\epsilon}\left(\eta e^{i \bar{\omega}}\right)$ can be expressed as

$$
\mathcal{I}^{\epsilon}\left(\eta e^{i \bar{\omega}}\right)=\frac{1}{(2 \pi)^{l} \epsilon^{s / 2}|W| \operatorname{vol}(T)} \int_{y \in \mathbf{t}^{*}} d y e^{-<y, y>/ 2 \epsilon} Q^{\eta}(y)
$$

where $W$ is the Weyl group of $K$ and $Q^{\eta}(\cdot)$ is a piecewise polynomial function on $\mathbf{t}^{*}$ supported on cones each of which has its apex at $\mu_{T}(F)$ for some component $F$ of the fixed point set of $T$ on $M$. Here as before $s$ is the dimension of $K$, while $l$ is the dimension of the maximal torus $T$. For the definition of $Q^{\eta}$ see the statement of Theorem 7.1 of [20]: it is

$$
Q^{\eta}=\mathcal{D} F_{T}\left(\mathcal{D} \sigma^{\eta}\right)
$$

where

$$
\sigma^{\eta}(X)=\Pi_{*}\left(e^{i \omega} e^{i \mu(X)} \eta(X)\right),
$$

and $\Pi_{*}$ denotes the integral over $M$ while $F_{T}$ is the Fourier transform over t. Equivalently if $\left\{e_{j}\right\}$ is a basis for $\mathbf{t}$ so that an element of $\mathbf{t}$ is given as $y=\sum_{j} y_{j} e_{j}$, we may write

$$
Q^{\eta}(y)=\mathcal{D P}_{\mathcal{D}}\left(F_{T} \Pi_{*} \sigma^{\eta}\right)
$$

where we define the differential operator

$$
\mathcal{P}_{\mathcal{D}}=\prod_{\gamma>0} \sum_{j} \gamma\left(e_{j}\right) \frac{\partial}{\partial y_{j}}
$$


as a product over the positive roots $\gamma$ of $K$.

We shall need to introduce a set of (possibly degenerate) cones $\mathcal{C}=$ $\left\{C_{1}, \ldots, C_{d}\right\}$, each with apex at 0 , for which $\mathbf{t}^{*}$ is the union of $C \in \mathcal{C}$, the intersection of any two is contained in their boundaries and $Q^{\eta}$ is polynomial on a neighbourhood of 0 in each $C$. Let $Q_{0}^{\eta}$ be the piecewise polynomial function which is polynomial on each cone $C \in \mathcal{C}$ (each having its apex at 0 ) and which coincides with $Q^{\eta}$ near 0 . Let

$$
\mathcal{I}^{\epsilon}{ }_{0}\left(\eta e^{i \bar{\omega}}\right)=\frac{1}{(2 \pi)^{l} \epsilon^{s / 2}|W| \operatorname{vol}(T)} \int_{y \in \mathbf{t}^{*}}[d y] e^{-<y, y>/ 2 \epsilon} Q_{0}^{\eta}(y) .
$$

Then the argument of [20] Section 6 shows that there exist real numbers $\rho_{\beta}>0$ and functions $h_{\beta}: \mathbb{R}^{+} \rightarrow \mathbb{R}$ such that for some $N_{\beta} \geq 0$ the product $\epsilon^{N_{\beta}} h_{\beta}(\epsilon)$ remains bounded as $\epsilon \rightarrow 0^{+}$and

$$
\left|\mathcal{I}^{\epsilon}\left(\eta e^{i \bar{\omega}}\right)-\mathcal{I}_{0}^{\epsilon}\left(\eta e^{i \bar{\omega}}\right)\right| \leq \sum_{\beta} e^{-\rho_{\beta} / 2 \epsilon} h_{\beta}(\epsilon)
$$

In Section 8 of [20] a residue formula is given for $\mathcal{I}_{0}^{\epsilon}\left(\eta e^{i \bar{\omega}}\right)$ in the case when 0 is a regular value of the moment map $\mu$. In this case $n_{0} \mathcal{I}_{0}^{\epsilon}\left(\eta e^{i \bar{\omega}}\right)$ is equal to $\eta_{0} e^{i \omega+\epsilon \Theta}[M / / K]$, where $\Theta \in H^{*}(M / / G)$ was defined at (1.3) and $n_{0}$ is the order of the stabilizer of a generic point of $\mu^{-1}(0)$, and thus $I_{0}^{\epsilon}\left(\eta e^{i \bar{\omega}}\right)$ is a polynomial function of $\epsilon$. The proof of Theorem 8.1 of [20] can be modified to obtain a formula for $\mathcal{I}_{0}^{\epsilon}\left(\eta e^{i \bar{\omega}}\right)$ in the general case when it may not be a polynomial in $\epsilon$ (see Example 9.7 below).

If $F \in \mathcal{F}$ is a component of the fixed point set of the maximal torus of $T$ acting on $M$ let $\beta_{F, j}$ (for $j \in J_{F}$ ) be the weights of the action of $T$ on the normal bundle to $F$ in $M$. We choose a connected component $\Lambda$ of the set of $\xi \in \mathbf{t}$ for which $\beta_{F, j}(\xi) \neq 0$ for all $F$ and $j$; we then adjust the signs of the $\beta_{F, j}$ (for all $F$ and $j$ ) in such a way that $\beta_{F, j}(\xi)>0$ for all $\xi \in \Lambda$ (see [16]).

We can then define $\operatorname{res}^{\Lambda}(h(X)[d X])$ as in [20] Section 8 when $h(X)$ is of the form

$$
h_{\lambda}(X)=\frac{q(X) e^{i \lambda(X)}}{\beta_{1}(X) \ldots \beta_{N}(X)},
$$

where $q(X)$ is a polynomial in $X \in \mathbf{t}$ while $\beta_{1}, \ldots, \beta_{N} \in \mathbf{t}^{*}$ all lie in the dual cone of $\Lambda$ and $\lambda \in \mathbf{t}^{*}$ does not lie in any cone of dimension at most $l-1$ spanned by a subset of $\left\{\beta_{1}, \ldots, \beta_{N}\right\}$. By [21] Proposition 3.2 it is uniquely determined by the following properties:

i) If $\left\{\beta_{1}, \ldots, \beta_{N}\right\}$ does not span $\mathbf{t}^{*}$ as a vector space then $\operatorname{res}^{\Lambda}\left(h_{\lambda}(X)[d X]\right)=0$.

ii) $\operatorname{res}^{\Lambda}\left(h_{\lambda}(X)[d X]\right)=\sum_{m \geq 0} \lim _{s \rightarrow 0+} \operatorname{res}^{\Lambda}\left(\frac{(i \lambda(X))^{m}}{m !} h_{s \lambda}(X)[d X]\right)$.

iii) If $q(X)=X_{1}^{j_{1}} \ldots X_{l}^{j_{l}}$ then the $\operatorname{limit}_{\lim _{s \rightarrow 0+}} \operatorname{res}^{\Lambda}\left(h_{s \lambda}(X)[d X]\right)$ is 0 unless $N=l+j_{1}+\ldots .+j_{l}$.

iv) If $q(X)=1$ and $N=l$ and $\left\{\beta_{1}, \ldots, \beta_{l}\right\}$ is a basis for $\mathbf{t}^{*}$ then $\operatorname{res}^{\Lambda}\left(h_{\lambda}(X)[d X]\right)=$ 0 unless $\lambda=\lambda_{1} \beta_{1}+\ldots+\lambda_{l} \beta_{l}$ where $\lambda_{j}>0$ for each $j$, and if this is the case 
then $\operatorname{res}^{\Lambda}\left(h_{\lambda}(X)[d X]\right)=|\operatorname{det} \bar{\beta}|^{-1}$, where $\bar{\beta}$ is an $l \times l$ matrix whose columns are the coordinates of $\beta_{1}, \ldots, \beta_{l}$ with respect to any orthonormal basis of $\mathbf{t}$.

Finally in order to remove the restriction on $\lambda$ we choose $\rho \in \mathbf{t}^{*}$ such that $-\rho$ lies in the dual cone of $\Lambda$ and define

$$
\operatorname{res}^{\rho, \Lambda}\left(h_{\lambda}(X)[d X]\right)=\lim _{s \rightarrow 0+} \operatorname{res}^{\Lambda}\left(h_{\lambda+s \rho}(X)[d X]\right)
$$

Except for the additional factors of $i$ discussed in Remark 3.4, when applied to suitable meromorphic differential forms on the complexified Lie algebra of the torus, $\operatorname{res}^{\rho, \Lambda}$ gives the multivariable residue which appeared in Section 3.

If $F \in \mathcal{F}$ we define a cone $C(F)$ in $\mathbf{t}$, with apex at $\mu_{T}(F)$, by

$$
C(F)=\left\{\mu_{T}(F)-\sum_{j} s_{j} \beta_{F, j}: s_{j} \geq 0\right\}
$$

where the $\beta_{F, j}$ are the weights of the action of $T$ on the normal bundle to $F$ with adjusted signs as above. By subdividing the cones $C \in \mathcal{C}$ if necessary, we can assume that for each $F \in \mathcal{F}$ and each $C \in \mathcal{C}$, either there is a neighbourhood of 0 in $C$ which is contained in $C(F)$ or there is a neighbourhood of 0 in $C$ which does not meet the interior of $C(F)$. Then if $F \in \mathcal{F}$ we let $\mathcal{C}_{F}$ be the set of $C \in \mathcal{C}$ such that there is a neighbourhood of 0 in $C$ which is contained in $C(F)$.

Theorem 43 If $\eta \in H_{K}^{*}(M)$ then $\mathcal{I}_{0}^{\epsilon}\left(\eta e^{i \bar{\omega}}\right)$ is equal to

$\frac{A_{K}}{\epsilon^{s / 2}} \sum_{F \in \mathcal{F}} \sum_{C \in \mathcal{C}_{F}} \int_{y \in C}[d y] \mathcal{D}(y) e^{-<y, y>/ 2 \epsilon} \mathcal{P}_{C} \operatorname{res}^{\rho, \Lambda}\left(\mathcal{D}(X) \int_{F} \frac{i_{F}^{*}\left(\eta(X) e^{i \omega}\right)}{e_{F}(X)} e^{i<\mu(F)-y, X>}\right)$

where the constant $A_{K}$ is given by

$$
A_{K}=\frac{i^{l}(2 \pi)^{-l / 2}}{|W| \operatorname{vol}(T)}
$$

Here $e_{F}$ denotes, as before, the equivariant Euler class of the normal bundle to $F$, and if $f$ is a piecewise polynomial function which is polynomial on a neighbourhood of 0 in the cone $C$, then $\mathcal{P}_{C}(f)$ denotes the polynomial which is equal to $f$ on a neighbourhood of 0 in $C$.

Proof: The proof is a straightforward modification of [20, Sections 4 and 8. By (9.1), we have

$$
\begin{aligned}
& I^{\epsilon}\left(\eta e^{i \bar{\omega}}\right)=\frac{1}{(2 \pi)^{s} \operatorname{vol} K} \int_{X \in \mathbf{k}}[d X] e^{-\epsilon|X|^{2} / 2} \int_{M} \eta(X) e^{i \bar{\omega}(X)} \\
& =\frac{1}{(2 \pi)^{l}|W| \operatorname{vol} T} \int_{X \in \mathbf{t}}[d X] e^{-\epsilon|X|^{2} / 2} \mathcal{D}(X)^{2} \int_{M} \eta(X) e^{i \bar{\omega}(X)}
\end{aligned}
$$


By Parseval's theorem this becomes

$I^{\epsilon}\left(\eta e^{i \bar{\omega}}\right)=\frac{1}{(2 \pi)^{l} \epsilon^{s / 2}|W| \operatorname{vol} T} \int_{y \in \mathbf{t}^{*}}[d y] \mathcal{D}(y) e^{-|y|^{2} / 2 \epsilon} F_{T}\left(\mathcal{D}(X) \int_{M} \eta(X) e^{i \omega} e^{i \mu(X)}\right)$,

where $F_{T}$ denotes the Fourier transform over $\mathbf{t}$. We now expand the integral over $M$ using the abelian localization theorem, to get a sum of terms

$$
\sum_{F \in \mathcal{F}} \int_{F} \frac{i_{F}^{*}\left(\eta(X) e^{i \omega}\right)}{e_{F}(X)} e^{i\langle\mu(F), X\rangle}
$$

each corresponding to a component $F$ of the fixed point set of the action of $T$ (see for example [16]). The Fourier transform can thus be expressed as a sum over $\mathcal{F}$ such that the term corresponding to $F \in \mathcal{F}$ is a piecewise polynomial function supported on a cone with apex at $\mu_{T}(F)$. Decomposing $\mathbf{t}^{*}$ into cones $C$ with apex at 0 as above, we find that the integrand on each cone $C$ is $e^{-|y|^{2} / 2 \epsilon}$ times a piecewise polynomial function $p_{C}$. The functions

$$
\mathcal{D}(y) \operatorname{res}^{\rho, \Lambda}\left(\mathcal{D}(X) \int_{F} \frac{i_{F}^{*}\left(\eta(X) e^{i \omega}\right)}{e_{F}(X)} e^{i\langle\mu(F)-y, X\rangle}\right)
$$

are also piecewise polynomial, and we can assume that they are also polynomial on a neighbourhood of 0 in each of the cones $C \in \mathcal{C}$. To define $I_{0}^{\epsilon}\left(\eta e^{i \bar{\omega}}\right)$, we replace $p_{C}$ by the polynomial $p_{C}^{0}$ which equals $p_{C}$ in a neighbourhood of 0 in $C$, which by the argument of [20] Section 8 is

$$
(2 \pi)^{l / 2} i^{l} \mathcal{D}(y) \mathcal{P}_{C} \mathrm{res}^{\rho, \Lambda}\left(\mathcal{D}(X) \int_{F} \frac{i_{F}^{*}\left(\eta(X) e^{i \omega}\right)}{e_{F}(X)} e^{i\langle\mu(F)-y, X\rangle}\right) .
$$

This gives us the formula in the statement of the theorem.

Remark 44 If the condition that $C \in \mathcal{C}_{F}$ (in other words that a neighbourhood of 0 in $C$ lies in $C(F)$ ) guarantees that the residue

$$
\operatorname{res}^{\rho, \Lambda}\left(\mathcal{D}(X) \int_{F} \frac{i_{F}^{*}\left(\eta(X) e^{i \omega}\right)}{e_{F}(X)} e^{i\langle\mu(F)-y, X\rangle}\right)
$$

is a polynomial function of $y$ on $C$ (not merely piecewise polynomial), then we can omit the expression $\mathcal{P}_{C}$ from (9.6).

Remark 45 If $F \in \mathcal{F}$ is such that $\mu_{T}(F)$ does not lie on a wall through 0 (or a wall such that the affine hyperplane spanned by the wall passes through $0)$, then 0 does not lie on the boundary of the cone $C(F)$, and hence either $\mathcal{C}_{F}=\emptyset$ or $\mathcal{C}_{F}=\mathcal{C}$. If $\mathcal{C}_{F}=\emptyset$ then $F \notin \mathcal{F}_{+}$and $F$ contributes zero to the expression for $\mathcal{I}_{0}^{\epsilon}\left(\eta e^{i \bar{\omega}}\right)$ in Theorem 9.1. If $\mathcal{C}_{F}=\mathcal{C}$, so that $F \in \mathcal{F}_{+}$, then the contribution of $F$ can be written as an integral over $\cup_{C \in \mathcal{C}} C=\mathbf{t}$ and by the arguments of [20] Section 8 it is given by the same formula

$$
C_{K} \operatorname{res}^{\rho, \Lambda}\left(\mathcal{D}(X)^{2} \int_{F} \frac{i_{F}^{*}\left(\eta e^{i \bar{\omega}}\right)(X) e^{-\epsilon<X, X>/ 2}}{e_{F}(X)}[d X]\right)
$$

as in the case when 0 is a regular value of $\mu$. 
Example 46 If $l=1$ and there are two cones (both half lines) then when calculating $\mathcal{I}_{0}^{\epsilon}\left(\eta e^{i \bar{\omega}}\right)$ we need integrals of the form

$$
\int_{y \in C} e^{-<y, y>/ 2 \epsilon} y^{j} d y= \pm \int_{0}^{\infty} e^{-y^{2} / 2 \epsilon} y^{j} d y .
$$

Ignoring the sign, this can be evaluated as

$$
\begin{gathered}
{\left[-\epsilon e^{-y^{2} / 2 \epsilon} y^{j-1}\right]_{0}^{\infty}+\epsilon(j-1) \int_{0}^{\infty} e^{-y^{2} / 2 \epsilon} y^{j-2} d y} \\
=\epsilon(j-1) \int_{0}^{\infty} e^{-y^{2} / 2 \epsilon} y^{j-2} d y
\end{gathered}
$$

if $j>1$. By induction this equals

$$
\begin{gathered}
\epsilon^{(j-1) / 2}(j-1)(j-3) \ldots 4 \cdot 2 \int_{0}^{\infty} e^{-y^{2} / 2 \epsilon} y d y \\
=\epsilon^{(j+1) / 2}(j-1)(j-3) \ldots 4 \cdot 2
\end{gathered}
$$

if $j$ is odd, and if $j$ is even it is

$$
\begin{gathered}
\epsilon^{j / 2}(j-1)(j-3) \ldots 3 \cdot 1 \int_{0}^{\infty} e^{-y^{2} / 2 \epsilon} d y \\
=\sqrt{\frac{\pi}{2}} \epsilon^{(j+1) / 2}(j-1)(j-3) \ldots 3 \cdot 1 .
\end{gathered}
$$

Thus we expect that $\sqrt{\epsilon}$ will appear in the answer and $\mathcal{I}_{0}^{\epsilon}\left(\eta e^{i \bar{\omega}}\right)$ will not in general be a polynomial in $\epsilon$.

Remark 47 By subdividing the cones $C \in \mathcal{C}$ if necessary, we can assume that each $C \in \mathcal{C}$ is of the form

$$
C=\left\{s_{1} b_{1}+\ldots+s_{l} b_{l}: s_{1}, \ldots, s_{l} \in \mathbb{R}, s_{1} \geq 0, \ldots, s_{m} \geq 0\right\}
$$

for some basis $b_{1}, \ldots, b_{l}$ of $\mathbf{t}$ and some $m \in\{0, \ldots, l\}$. Then the formula of Theorem 9.1 can be expressed as a linear combination (whose coefficients are independent of $\epsilon$ ) of integrals of the form

$$
\int_{y \in C}[d y] p(y) e^{-\langle y, y\rangle / 2 \epsilon}
$$

where $p(y)$ is a polynomial function of $y \in \mathbf{t}$. Changing coordinates using the basis $b_{1}, \ldots, b_{l}$ of $\mathbf{t}$ gives us integrals of the form

$$
\int_{y \in\left(\mathbb{R}^{+}\right)^{m} \times \mathbb{R}^{l-m}}[d y] P(y) e^{-\langle y, y\rangle / 2 \epsilon}
$$

where $P(y)$ is a polynomial function of $y \in \mathbb{R}^{l}$ and $\langle$,$\rangle is an inner product on$ $\mathbb{R}^{l}$. Using induction on $l$ and calculations similar to those in Example 46] it follows that $\mathcal{I}_{0}^{\epsilon}\left(\eta e^{i \bar{\omega}}\right)$ is always a polynomial in $\sqrt{\epsilon}$, although not necessarily a polynomial in $\epsilon$. 
Example 48 Consider the case we have looked at a number of times (Examples $2.4,3.2,4.2)$ when $G=S L(2)$ acts on $M=\left(\mathbf{P}_{1}\right)^{n}$ with $n$ even (cf. also Section 9 of [20]). The fixed points of the action of the maximal torus $T$ of $K$ are the $n$-tuples of points $\left(x_{1}, \ldots, x_{n}\right)$ in $\mathbf{P}_{1}$ such that each $x_{j}$ is either 0 or $\infty$, which we index by sequences $\underline{\delta}=\left(\delta_{1}, \ldots, \delta_{n}\right)$ where $\delta_{j}=1$ if $x_{j}=0$ and $\delta_{j}=-1$ if $x_{j}=\infty$. The value of the moment map for such a point is $\sum_{j=1}^{n} \delta_{j}$. Recall that $H^{*}(M)$ has $n$ generators of degree 2 , and the equivariant cohomology $H_{T}^{*}(M)$ is generated by lifts $\xi_{i}(i=1, \ldots, n)$ of these together with one additional generator $\zeta$ of degree 2 , subject to the relations $\left(\xi_{j}\right)^{2}=\zeta^{2}$, while $H_{K}^{*}(M)$ is generated by the $\xi_{j}$ and $\zeta^{2}$ subject to the same relations. Then if $\eta=q\left(\xi_{1}, \ldots, \xi_{n}, \zeta^{2}\right) \in H_{K}^{*}(M)$ we have from Theorem 9.1 that

$$
\begin{gathered}
\mathcal{I}_{0}^{\epsilon}\left(\eta e^{i \bar{\omega}}\right)=\frac{A_{K}}{\epsilon^{s / 2}}\left(\sum_{\delta_{1}+\ldots+\delta_{n}>0}\left(\int_{-\infty}^{0} d y e^{-y^{2} / 2 \epsilon} y \mathcal{P}_{-\mathbb{R}^{+}}+\int_{0}^{\infty} d y e^{-y^{2} / 2 \epsilon} y \mathcal{P}_{\mathbb{R}^{+}}\right)\right. \\
\left.\quad+\sum_{\delta_{1}+\ldots+\delta_{n}=0} \int_{-\infty}^{0} d y e^{-y^{2} / 2 \epsilon} y \mathcal{P}_{-\mathbb{R}^{+}}\right) \times \\
\operatorname{res}_{X=0}^{+}\left(\frac{1}{X^{n-1}} e^{i X\left(\sum_{j} \delta_{j}\right)} e^{-i y X}\left(\prod_{j} \delta_{j}\right) q\left(\delta_{1} X, \ldots, \delta_{n} X, X^{2}\right)\right)
\end{gathered}
$$

where $A_{K}$ is the constant defined in the statement of Theorem 9.1, and for a rational function $R(X), \operatorname{res}_{X=0}^{+}\left(e^{i \mu X} R(X)\right)$ is the coefficient of $1 / X$ in the Taylor expansion if $\mu \geq 0$ and 0 otherwise.

An easy calculation shows that the factors $\mathcal{P}_{ \pm \mathbb{R}}+\operatorname{res}_{X=0}^{+}$may simply be replaced by $\operatorname{res}_{X=0}$. The contribution of any fixed point indexed by $\{\underline{\delta}\}$ such that $\sum_{j} \delta_{j}=0$ is

$$
A_{K} \epsilon^{-3 / 2} \operatorname{res}_{X=0}\left(\int_{-\infty}^{0} \frac{(-1)^{n / 2} y}{X^{n-1}} q\left(\delta_{1} X, \ldots, \delta_{n} X, X^{2}\right) e^{-y^{2} / 2 \epsilon} e^{-i y X} d y\right) .
$$

Thus we see from the calculations in Example 46 that for some choices of $q$ the individual contributions of the $F$ with $\mu_{T}(F)=0$ will involve odd powers of $\sqrt{\epsilon}$, although an argument from symmetry shows that $\mathcal{I}_{0}^{\epsilon}\left(\eta e^{i \bar{\omega}}\right)$ is in fact a polynomial in $\epsilon$ in this example.

Example 49 As our final example consider the linear action of $G=\mathbb{C}^{*}$ on $M=\mathbb{P}_{n}$ with distinct weights $r_{0}, \ldots, r_{n} \in \mathbb{Z}$. Then $H_{T}^{*}(M)$ is generated by two equivariant cohomology classes $\xi$ and $\zeta$ of degree two subject to the relation

$$
\prod_{j=0}^{n}\left(\xi-r_{j} \zeta\right)=0
$$

The moment map for the action of $T=S^{1}$ on $M$ is given up to a constant by

$$
\mu\left[x_{0}, \ldots, x_{n}\right]=\frac{r_{0}\left|x_{0}\right|^{2}+\ldots+r_{n}\left|x_{n}\right|^{2}}{\left|x_{0}\right|^{2}+\ldots+\left|x_{n}\right|^{2}}
$$


Suppose now that $r_{0}=0$ but that $r_{j} \neq 0$ for $j>0$. Then if $\eta=q(\xi, \zeta) \in$ $H_{T}^{*}(M)$ we have from Theorem 9.1 that

$$
\begin{gathered}
\mathcal{I}_{0}^{\epsilon}\left(\eta e^{i \bar{\omega}}\right)=\frac{A_{K}}{\sqrt{\epsilon}} \int_{-\infty}^{0} d y \operatorname{res}_{X=0}\left(\frac{e^{-y^{2} / 2 \epsilon} e^{-i y X}}{\prod_{k \neq 0}\left(r_{k} X-r_{0} X\right)} q(0, X)\right. \\
\left.+\sum_{j: r_{j}>0} \int_{-\infty}^{\infty} d y \operatorname{res}_{X=0} \frac{e^{i r_{j} X} e^{-y^{2} / 2 \epsilon} e^{-i y X}}{\prod_{k \neq j}\left(r_{k} X-r_{j} X\right)} q\left(r_{j} X, X\right)\right) .
\end{gathered}
$$

The contribution of each $j>0$ to this expression is a polynomial in $\epsilon$, but if $q(0, X)=X^{N}$ then

$$
\operatorname{res}_{X=0} \int_{-\infty}^{0} d y \frac{e^{-y^{2} / 2 \epsilon} e^{-i y X}}{\prod_{k \neq 0}\left(r_{k} X-r_{0} X\right)} q(0, X)=\int_{-\infty}^{0} d y \frac{e^{-y^{2} / 2 \epsilon}(-i y)^{n-N-2}}{\prod_{k \neq 0}\left(r_{k}-r_{0}\right)}
$$

which is a nonzero constant multiple of $\epsilon^{(n-N-2) / 2}$. Thus if $n-N$ is odd then $\mathcal{I}_{0}^{\epsilon}\left(\eta e^{i \bar{\omega}}\right)$ is not a polynomial in $\epsilon$.

\section{References}

[1] M.F. Atiyah, Convexity and commuting Hamiltonians, Bull.London Math. Soc. 14 (1982) 1-15.

[2] M.F. Atiyah, R. Bott, The Yang-Mills equations over Riemann surfaces. Philos. Trans. Roy. Soc. London Ser. A 308 (1983), no. 1505,523-615.

[3] M.F. Atiyah, R. Bott, The moment map and equivariant cohomology, Topology 23 (1984) 1-28.

[4] A.A. Beilinson, J. Bernstein, P. Deligne, Faisceaux pervers, Astérisque 100 (1982), 5-171.

[5] N. Berline, E. Getzler, M. Vergne, Heat Kernels and Dirac Operators, Springer-Verlag (Grundlehren vol. 298), 1992.

[6] N. Berline, M. Vergne, Zéros d'un champ de vecteurs et classes caractéristiques équivariantes, Duke Math. J. 50 (1983) 539-549.

[7] N. Berline, M. Vergne, The equivariant index and Kirillov's character formula, Amer. J. Math. 107 (1985) 1159-1190.

[8] T. Bröcker, T. Tom Dieck, Representations of Compact Lie Groups, Springer-Verlag, 1985.

[9] H. Cartan, Notions d'algèbre différentielle; applications aux variétés où opère un groupe de Lie, in Colloque de Topologie (C.B.R.M., Bruxelles, 1950) 15-27; La transgression dans un groupe de Lie et dans un fibré principal, op. cit., 57-71. 
[10] J.J Duistermaat, G. Heckman, On the variation in the cohomology of the symplectic form of the reduced phase space, Invent. Math. 69 (1982) 259-268; Addendum, 72 (1983) 153-158.

[11] M. Goresky and R. MacPherson, Intersection homology theory, Topology 19 (1980), 135-162.

[12] M. Goresky and R. MacPherson, Intersection homology II, Invent. Math. 71 (1983), 77-129.

[13] P. Griffiths, J. Harris, Principles of algebraic geometry, WileyInterscience, 1978.

[14] V. Guillemin, J. Kalkman, The Jeffrey-Kirwan localization theorem and residue operations in equivariant cohomology. J. Reine Angew.Math. 470 (1996), 123-142.

[15] V. Guillemin, S. Sternberg, Convexity properties of the moment mapping I and II, Invent. Math. 67 (1982) 515-538 and 77 (1984) 533-546.

[16] V. Guillemin, E. Lerman, S. Sternberg, On the Kostant multiplicity formula, J. Geom. Phys. 5 (1988) 721-750 (1988).

[17] V. Guillemin, S. Sternberg, Symplectic Techniques in Physics, Cambridge University Press, 1984.

[18] H. Hironaka, Resolution of singularities of an algebraic variety over a field of characteristic zero. I, II. Ann. of Math. (2) 79 (1964)109-203; ibid. (2) 791964 205-326.

[19] L.C. Jeffrey, Y.H. Kiem, F.C. Kirwan, J. Woolf, Intersection pairings on singular moduli spaces of bundles over a Riemann surface, in preparation.

[20] L.C. Jeffrey, F.C. Kirwan, Localization for nonabelian group actions, Topology 34 (1995) 291-327.

[21] L.C. Jeffrey, F.C. Kirwan, Localization and the quantization conjecture, Topology 36 (1997) 647-693.

[22] L.C. Jeffrey, F.C. Kirwan, Intersection theory on moduli spaces of holomorphic bundles of arbitrary rank on a Riemann surface, Ann. Math. 148 (1998) 109-196.

[23] Y.-H. Kiem, Intersection cohomology of quotients of nonsingular varieties, Yale preprint (1999), math.AG/0101254.

[24] Y.-H. Kiem, J. Woolf, The cosupport axiom, equivariant cohomology and the intersection cohomology of certain symplectic quotients, preprint math.AG/0101255. 
[25] F.C. Kirwan, Cohomology of Quotients in Symplectic and Algebraic Geometry, Princeton University Press, 1984.

[26] F.C. Kirwan, Partial desingularisations of quotients of nonsingular varieties and their Betti numbers, Ann. Math. 122 (1985), 41-85.

[27] F.C. Kirwan, Rational intersection cohomology of quotient varieties, Invent. Math 86 (1986), 471-505.

[28] D. Luna, Sur les orbites fermés des groupes algébriques réductifs, Invent. Math. 16 (1972), 1-5.

[29] S.K. Martin, Symplectic geometry and gauge theory, Oxford D.Phil. thesis, 1997.

[30] S.K. Martin, Symplectic quotients by a nonabelian group and by its maximal torus, math.SG/0001002 Annals of Mathematics, to appear.

[31] S.K. Martin, Transversality theory, cobordisms, and invariants of symplectic quotients, math.SG/0001001, Annals of Mathematics, to appear.

[32] E. Meinrenken and R. Sjamaar, Singular reduction and quantization, Topology 38 (1999), 699-762.

[33] G. Mostow, On a conjecture of Montgomery, Ann. Math. 65 (1957), 513-516.

[34] D. Mumford, J. Fogarty, F. Kirwan, Geometric invariant theory, 3rd edition, Springer-Verlag, 1994.

[35] P.E. Newstead, Introduction to moduli problems and orbit spaces, Tata Institute Lectures 51, Springer-Verlag, 1978.

[36] P.-E. Paradan, The moment map and equivariant cohomology with generalized coefficients, Topology 39 (2000), 401-444.

[37] S. Tolman and J. Weitsman, The cohomology rings of abelian symplectic quotients, preprint math.DG/9807173.

[38] M. Vergne, A note on the Jeffrey-Kirwan-Witten localisation formula. Topology 35 (1996), 243-266.

[39] E. Witten, Two dimensional gauge theories revisited, preprint hep-th/9204083 J. Geom. Phys. 9 (1992) 303-368.

[40] J. Woolf, Some Topological Invariants of Singular Symplectic Quotients, D.Phil. thesis, Oxford, 1999.

[41] J. Woolf, The decomposition theorem and the intersection cohomology of quotients in algebraic geometry, preprint math.AG/0110137 (2000). 